\title{
A PLASMA EXPANSION MODEL BASED ON THE FULL EULER-POISSON SYSTEM
}

\author{
P. Crispel \\ CNES Centre de Toulouse, 18 av. Ed. Belin, 31401 Toulouse cedex 4, FRANCE. \\ ONERA Centre de Toulouse, 2 av. Ed. Belin, 31055 Toulouse cedex 4, FRANCE. \\ MIP, Univ. P. Sabatier, 118 rte de Narbonne, 31062 Toulouse cedex 4, FRANCE. \\ crispel@mip.ups-tlse.fr \\ P. Degond, M. H. Vignal ${ }^{\dagger}$ \\ MIP, Univ. P. Sabatier, 118 rte de Narbonne, 31062 Toulouse cedex 4, FRANCE. \\ *degond@mip.ups-tlse.fr \\ †mhvignal@mip.ups-tlse.fr
}

\begin{abstract}
In this paper, we consider a quasi-neutral plasma expanding in the vacuum gap separating two electrodes. During the expansion, some particles are emitted from the plasmavacuum interface and form a beam in the vacuum. Starting from the two-fluid full EulerPoisson model, we derive an asymptotic model. This asymptotic model consists of a quasi-neutral model in the plasma region, a Child-Langmuir law in the beam region and connection relations at the plasma-beam interface. For this model, we propose a numerical scheme which accounts for the motion of the plasma-beam interface and is much more efficient than the resolution of the original two-fluid Euler-Poisson problem. We demonstrate the efficiency of the model by means of numerical simulations for two different one-dimensional test cases.

Keywords: Expanding plasma, full-Euler-Poisson model, quasi-neutral limit, ChildLangmuir law, travelling-wave analysis, Bohm sheath criterion.
\end{abstract}

AMS Subject Classification: 82D10,76W05,76X05,76N10,76N20,76L05

\section{Introduction}

In this paper, which is a continuation of earlier works ${ }^{10,13}$, we are concerned with the modeling of a quasi-neutral plasma expansion in the vacuum gap separating two electrodes. Such a problem arises in the study of high current diodes mentioned in Ref. 27, in electrical discharges on satellite solar panels, see Ref. 6 or in high current injectors for ion accelerators, see Ref. 18. These accelerators are used, among other applications, to produce isotopes for medical applications. In the case of the high current diode application, the plasma is used to increase the extracted current as compared with conventional plane diodes. For the solar panel problem,

${ }^{*}$ Corresponding author 
our model can be used for the transition from the primary discharge to the electric arc, see Ref. 17 for more details.

In all these problems, the physical process is the following: a quasi-neutral plasma, considered as fully ionized and constituted of only one ion species is injected from an electrode namely the anode in the case of high current injectors and the cathode in the cases of high current diodes and of arc phenomena on satellites. This plasma undergoes a thermal expansion in the gap separating the electrodes. But, due to the applied potential difference, some particles (the ions in the case of high current injectors and the electrons in the cases of high current diodes and of arc phenomena on satellites) are emitted from the plasma-vacuum interface into the gap. These particles form a non quasi-neutral beam in the vacuum.

The description of such a problem can be performed with the two-fluid full Euler-Poisson model. This model consists of the full Euler system for each species (the ions and the electrons) coupled with the Poisson equation for the electric potential. By full Euler model, we mean the complete set of mass, momentum and energy equations, as opposed to the isentropic approximation used in Ref 10, 13 . Unfortunately, this system is subject to severe numerical constraints in the quasineutral zones. Indeed, in plasmas, local charge imbalances can occur only at the scale of the Debye length, see Ref. 4 and Ref. 20. Due to these local charge imbalances and to electric restoring forces the particles oscillates around their equilibrium positions. The period of the electron oscillations is called the plasma period. In order to avoid numerical instabilities, the scheme used for the discretization of the two-fluid Euler-Poisson model must resolve the scale of the plasma period. But, in quasineutral plasmas, the Debye length and the plasma period are very small parameters. Then, the numerical simulations of the two-fluid Euler-Poisson model is virtually impossible in dimensions greater than one.

There are two possible ways to overcome this limitation. The first way consists in finding an asymptotically stable discretization, i.e. a scheme which does not require the resolution of the plasma period. Such a scheme has been developed in Ref. 11 for the two-fluid Euler-Poisson system in the isentropic case, see also Ref. 5, 7, 24, 25. The second way, that we choose to explore here, consists in finding an asymptotic model in the quasi-neutral regions. In Ref. 10 and Ref. 13, this study has been done in the isentropic case. Here, we extend this method when full Euler models with energy conservation laws are considered.

Starting from the two-fluid Euler-Poisson system and performing a formal quasineutral limit, we derive a quasi-neutral model with a non vanishing current for the plasma region. This model is a non conservative hyperbolic system. But in the considered applications, that is high current diodes and arc phenomena on satellites, the fluid quantities are regular. Then, the non conservativity of the model is not a problem. Rigorous quasi-neutral limits of the Euler-Poisson system have been investigated in Ref. 8, 22, 26, 29, mostly in the current-free case.

In the beam region, only one species is present and the quasi-neutral model is no more valid. Then, introducing a new scaling we derive a Child-Langmuir law. This 
model consists of a stationary Euler-Poisson model with a pressureless momentum equation, see Ref. 21 and Ref. 1, 12, 16 for the mathematical aspects.

Then, the domain is divided in two regions: the quasi-neutral region, i.e. the plasma bubble and the non quasi-neutral beam region. Therefore two different models coexist in different parts of the domain and we must reconnect them at the plasma-beam interface. Furthermore, due to the plasma expansion between the electrodes, we must describe the dynamics of the interface between the plasma and the beam. To this aim, we introduce a transmission problem which consists in analyzing the transition region around the interface as a traveling-wave solution of the two-fluid Euler-Poisson. This traveling-wave solution is connecting the quasineutral model on the plasma side to the Child-Langmuir model on the beam side. Here, this problem reduces to the isentropic transmission problem. Then using the results proved in Ref. 13, we get convenient transmission relations between the quasi-neutral and the Child-Langmuir model.

We propose a numerical scheme for the discretization of the asymptotic model. In order to approximate the interface velocity, we solve a particular Riemann problem at the plasma beam interface. This problem consists of a fluid-vacuum Riemann problem but with a non vanishing pressure in the vacuum. This is due to the fact that emitted particles from the interface exert a pressure onto the plasma. In order to validate our asymptotic model, we perform numerical simulations for two different one-dimensional test cases. The first test case is related to high current injectors for ion accelerators and the second test case to high current diode or arc phenomena on satellites.

\section{The physical problem and the two-fluid Euler-Poisson model}

We denote by $\bar{x}=\left(\bar{x}_{1}, \cdots, \bar{x}_{d}\right) \in \mathbb{R}^{d}(d=1,2$ or 3$)$ and $\bar{t}>0$ the space and time variables, and we consider two plane electrodes located at $\bar{x}_{1}=0$ and $\bar{x}_{1}=L$. Then the domain is given by $\Omega=[0, L] \times \mathbb{R}^{d-1}$. We set the origin of the potential at $\bar{x}_{1}=0$ and the potential at $\bar{x}_{1}=L$ is given by $\bar{\phi}_{L}$. This potential $\bar{\phi}_{L}$ can be positive or negative, depending on the considered device. Here for clarity, we suppose that $\bar{\phi}_{L}<0$. Then, the anode is located at $\bar{x}_{1}=0$ and the cathode at $\bar{x}_{1}=L$. The symmetric case, i.e. when $\bar{\phi}_{L}>0$, can be easily deduced from this work (see Ref. 9). In Section 5 we give numerical results for both cases.

We consider that a quasi-neutral plasma, constituted of one positively charged ion species and of electrons, is injected from a part of the anode. Due to thermal effects, it expands in the gap between the electrodes. Simultaneously, attracted by the negative potential of the cathode located at $\bar{x}_{1}=L$, some ions are emitted from the plasma-vacuum interface forming an ion beam in the vacuum. Then, the domain is divided in two zones: the quasi-neutral plasma region and the non quasi-neutral beam region, provided the transition region reduces to an interface.

We use a fluid description and the particles (the ions and the electrons) are described by their densities, $N_{i}$ and $N_{e}$, their velocities, $U_{i}$ and $U_{e}$, and their total 
energies, $W_{i}$ and $W_{e}$, where the quantities for ions are indexed by $i$ and those for electrons by $e$. The two-fluid Euler-Poisson model is valid in all the device, i.e. in the quasi-neutral and in the non quasi-neutral zones. It is constituted of the Euler systems for the ions and electrons coupled with the Poisson equation for the electric potential. It is written

$$
\begin{aligned}
& \left\{\begin{array}{l}
\partial_{\bar{t}} N_{i}+\nabla_{\bar{x}} \cdot\left(N_{i} U_{i}\right)=0 \\
m_{i}\left(\partial_{\bar{t}}\left(N_{i} U_{i}\right)+\nabla_{\bar{x}}\left(N_{i} U_{i} \otimes U_{i}\right)\right)+\nabla_{\bar{x}} P_{i}=-e N_{i} \nabla_{\bar{x}} \Phi \\
\partial_{\bar{t}} W_{i}+\nabla_{\bar{x}} \cdot\left(W_{i} U_{i}+P_{i} U_{i}\right)=-e N_{i} U_{i} \cdot \nabla_{\bar{x}} \Phi
\end{array}\right. \\
& \left\{\begin{array}{l}
\partial_{\bar{t}} N_{e}+\nabla_{\bar{x}} \cdot\left(N_{e} U_{e}\right)=0 \\
m_{e}\left(\partial_{\bar{t}}\left(N_{e} U_{e}\right)+\nabla_{\bar{x}}\left(N_{e} U_{e} \otimes U_{e}\right)\right)+\nabla_{\bar{x}} P_{e}=e N_{e} \nabla_{\bar{x}} \Phi \\
\partial_{\bar{t}} W_{e}+\nabla_{\bar{x}} \cdot\left(W_{e} U_{e}+P_{e} U_{e}\right)=e N_{e} U_{e} \cdot \nabla_{\bar{x}} \Phi
\end{array}\right.
\end{aligned}
$$

where $\nabla_{\bar{x}}=\left(\partial_{\bar{x}_{1}}, \cdots, \partial_{\bar{x}_{d}}\right), m_{i}$ and $m_{e}$ are the particle masses, and $e>0$ is the elementary charge. The pressure laws $P_{i}$ and $P_{e}$ are defined by

$$
W_{e}=\frac{P_{e}}{\gamma_{e}-1}+\frac{m_{e} N_{e}\left|U_{e}\right|^{2}}{2}, \quad W_{i}=\frac{P_{i}}{\gamma_{i}-1}+\frac{m_{i} N_{i}\left|U_{i}\right|^{2}}{2}
$$

where $\gamma_{i, e}>1$ are the ratios of specific heats. We recall that the temperatures are obtained with the relations $P_{i, e}=N_{i, e} k_{B} \bar{T}_{i, e}, k_{B}$ being the Boltzmann constant. The electric potential $\Phi$ is calculated with the Poisson equation given by:

$$
-\Delta_{\bar{x}} \Phi=\frac{e}{\varepsilon_{0}}\left(N_{i}-N_{e}\right)
$$

where $\varepsilon_{0}$ is the vacuum permittivity.

In plasmas the local charge imbalances take place at the scale of the Debye length (see Ref. 4 and Ref. 20), defined by

$$
\lambda_{D}=\left(\frac{\varepsilon_{0} k_{B} \bar{T}_{0}}{e^{2} N_{0}}\right)^{1 / 2},
$$

where $\bar{T}_{0}$ and $N_{0}$ are the typical scales of the temperature and of the density in the plasma. When such charge imbalances occur, the particles oscillate around their equilibrium position due to the electric restoring forces. The periods of these oscillations, denoted by $\tau_{e}$ for electrons and by $\tau_{i}$ for ions, are given by

$$
\tau_{e}=\lambda_{D} / U_{t h, e}, \quad \text { and } \quad \tau_{i}=\lambda_{D} / U_{t h, i},
$$

where $U_{t h, e}=\sqrt{k_{B} \bar{T}_{0} / m_{e}}$ and $U_{t h, i}=\sqrt{k_{B} \bar{T}_{0} / m_{i}}$ are respectively the electron and ion thermal velocities. Note that due to the large ion to electron mass ratio, the ion plasma period $\tau_{i}$ is much greater than the electron plasma period $\tau_{e}$ which is usually called plasma period. In quasi-neutral plasmas the Debye length and the plasma period are very small compared to the typical length and time of interest. As a consequence, charge imbalances are negligible and an equilibrium between positive and negative charges occurs in the plasma, leading to the approximation $n_{i} \approx n_{e}$. 
By contrast, in non-quasi-neutral plasmas, the Debye length and the typical length of interest and similarly the plasma period and the typical time of interest are of the same order of magnitude. In this case, it is no more possible to average out charge imbalances.

In practice, when standard explicit schemes are used they must resolve the plasma period in order to avoid instabilities, see Ref. 19. Then, the time step must satisfy

$$
\Delta t \leq \tau_{e}
$$

In quasi-neutral regime, this constraint is so penalizing that it is virtually impossible to perform two or three dimensional simulations. In order to overcome this limitation, we choose to establish an asymptotic model. This model is presented in the next section and consists of a quasi-neutral system with a non vanishing current for the plasma region, a Child-Langmuir law for the beam region and some transmission relations at the plasma-beam interface.

\section{The asymptotic model}

We begin with the derivation of the quasi-neutral model. We recall that this model is only valid in the quasi-neutral zones, i.e. the plasma region. Then, in Section 3.2, we introduce the Child-Langmuir model used for the description of the non quasineutral beam region. Finally, we present the plasma beam interface model connecting the quasi-neutral and the non quasi-neutral zones.

\subsection{The quasi-neutral model}

The quasi-neutral model is obtained from the two-fluid Euler-Poisson model (2.1)(2.4), passing formally to the limit $\lambda_{D} / L \rightarrow 0$. First, we study this formal limit, then, we present a one-fluid formulation of this model. This formulation is simpler in the one-dimensional case and gives precious qualitative informations on the quasineutral model. Finally, we precise the boundary conditions for the quasi-neutral model.

\subsubsection{The formal quasi-neutral limit}

We begin with the rescaling of the system (2.1)-(2.4). For characteristic scales, we choose $N_{0}$ for the densities, $\bar{T}_{0}$ for the temperatures and $U_{t h, i}=\sqrt{k_{B} \bar{T}_{0} / m_{i}}$ for the velocities. We recall that $N_{0}$ and $\bar{T}_{0}$ are the typical scales of the density and of the temperature in the plasma. We define the new variables $t=\bar{t} / t_{0}$ and $x=\bar{x} / L$ with $t_{0}=L / U_{t h, i}$. The new unknowns are given by $n_{i, e}=N_{i, e} / N_{0}, u_{i, e}=U_{i, e} / U_{t h, i}$, $w_{i, e}=W_{i, e} /\left(k_{B} \bar{T}_{0}\right), p_{i, e}=P_{i, e} /\left(N_{0} k_{B} \bar{T}_{0}\right), \phi=e \Phi /\left(k_{B} \bar{T}_{0}\right)$ and $T_{i, e}=\bar{T}_{i, e} / \bar{T}_{0}$. Inserting these new variables in (2.1)-(2.4), we obtain the rescaled two-fluid Euler- 
Poisson system for all $x \in \Omega=[0,1] \times \mathbb{R}^{d-1}$ and all $t>0$

$$
\begin{aligned}
& \partial_{t} n_{i, e}+\nabla \cdot\left(n_{i, e} n_{i, e}\right)=0, \\
& \partial_{t}\left(n_{i} u_{i}\right)+\nabla\left(n_{i} u_{i} \otimes u_{i}\right)+\nabla p_{i}=-n_{i} \nabla \phi, \\
& \varepsilon\left(\partial_{t}\left(n_{e} u_{e}\right)+\nabla n_{e} u_{e} \otimes u_{e}\right)+\nabla p_{e}=n_{e} \nabla \phi, \\
& \partial_{t} w_{i}+\nabla \cdot\left(\left(w_{i}+p_{i}\right) u_{i}\right)=-n_{i} u_{i} \cdot \nabla \phi, \\
& \partial_{t} w_{e}+\nabla \cdot\left(\left(w_{e}+p_{e}\right) u_{e}\right)=n_{e} u_{e} \cdot \nabla \phi, \\
& -\lambda^{2} \Delta \phi=n_{i}-n_{e},
\end{aligned}
$$

where

$$
\varepsilon=m_{e} / m_{i}, \quad \text { and } \quad \lambda=\lambda_{D} / L
$$

are respectively the mass ratio and the scaled Debye length. We recall that $\lambda_{D}$ is given by (2.5). The rescaled equations of state are written

$$
w_{i}=\frac{p_{i}}{\gamma_{i}-1}+\frac{n_{i}\left|u_{i}\right|^{2}}{2}, \quad w_{e}=\frac{p_{e}}{\gamma_{e}-1}+\frac{\varepsilon n_{e}\left|u_{e}\right|^{2}}{2} .
$$

At the beginning of the process, there is no plasma in the gap. Therefore, the initial conditions are given by $\left.n_{i}\right|_{t=0}=\left.n_{e}\right|_{t=0}=0$ in all the domain, and $\left.u_{i}\right|_{t=0},\left.u_{e}\right|_{t=0}$ are undefined. We assume that a quasi-neutral plasma is emitted from the anode with the same velocities for the two species. We set the origin of potential at the anode. Then, the boundary conditions for (3.1)-(3.6) are given by

$$
\begin{aligned}
& \left.n_{i}\right|_{x_{1}=0}=\left.n_{e}\right|_{x_{1}=0}=n_{0},\left.\quad u_{i}\right|_{x_{1}=0}=\left.u_{e}\right|_{x_{1}=0}=u_{0},\left.\quad T_{i}\right|_{x_{1}=1}=\left.T_{e}\right|_{x_{1}=1}=T_{0}, \\
& \left.n_{i}\right|_{x_{1}=1}=\left.n_{e}\right|_{x_{1}=1}=0, \\
& \left.\phi\right|_{x_{1}=0}=0,\left.\quad \phi\right|_{x_{1}=1}=\phi_{L},
\end{aligned}
$$

where $n_{0}, u_{0}$ and $T_{0}$ are respectively the rescaled density, velocity and temperature of the injected plasma and $\phi_{L}=e \bar{\phi}_{L} /\left(k_{B} \bar{T}_{0}\right)$ is the rescaled cathode potential. For a hyperbolic system, the number of boundary conditions which can be prescribed depends on the number of incoming characteristics. So, (3.9) and (3.10) should be understood as follows: suppose that we introduce a discretization of the rescaled two-fluid Euler-Poisson system by means of a Godunov scheme. Then, (3.9) and (3.10) will be used to compute the flux of the Riemann problems across the domain boundary.

Now, taking the formal quasi-neutral limit, $\lambda \rightarrow 0$, gives

$$
\begin{aligned}
& \partial_{t} n_{i, e}+\nabla \cdot\left(n_{i, e} n_{i, e}\right)=0, \\
& \partial_{t}\left(n_{i} u_{i}\right)+\nabla\left(n_{i} u_{i} \otimes u_{i}\right)+\nabla p_{i}=-n_{i} \nabla \phi, \\
& \varepsilon\left(\partial_{t}\left(n_{e} u_{e}\right)+\nabla n_{e} u_{e} \otimes u_{e}\right)+\nabla p_{e}=n_{e} \nabla \phi, \\
& \partial_{t} w_{i}+\nabla \cdot\left(\left(w_{i}+p_{i}\right) u_{i}\right)=-n_{i} u_{i} \cdot \nabla \phi, \\
& \partial_{t} w_{e}+\nabla \cdot\left(\left(w_{e}+p_{e}\right) u_{e}\right)=n_{e} u_{e} \cdot \nabla \phi, \\
& n_{i}=n_{e} .
\end{aligned}
$$


Note that the electric potential is now the Lagrange multiplier of the quasineutrality constraint (3.17). This constraint yields a divergence-free current. Indeed, taking the difference between the mass conservation equations (3.12) and taking into account the quasi-neutrality constraint (3.17), we obtain

$$
\partial_{t}\left(n_{i}-n_{e}\right)+\nabla \cdot j=\nabla \cdot j=\nabla \cdot\left(n_{i} u_{i}-n_{e} u_{e}\right)=0 .
$$

Like in the isentropic case (see Ref. 11) an explicit equation for the electric potential can be found. We take the divergence of the difference between (3.13) and (3.14). Using (3.18) into the result, we obtain the quasi-neutral potential equation

$$
-\nabla \cdot\left(\left(n_{i}+\frac{n_{e}}{\varepsilon}\right) \nabla \phi\right)=\nabla^{2}:\left(f_{i}-f_{e}\right)
$$

where the symbols $\nabla^{2}$ and : respectively denote the tensor of second order derivatives and the contracted product of two tensors. The scaled momentum fluxes are given by $f_{i}=n_{i} u_{i} \otimes u_{i}+p_{i} I d$ and $f_{e}=n_{e} u_{e} \otimes u_{e}+p_{e} / \varepsilon I d$.

Thus, the quasi-neutral model consists of (3.8), (3.12)-(3.16) and (3.19). It is formally equivalent to (3.8), (3.12)-(3.17) provided the quasi-neutrality is initially satisfied i.e. $n_{i}(t=0)=n_{e}(t=0)$. It is important to note that this asymptotic model is only valid in quasi-neutral regions i.e. in the regions where $\lambda$ is sufficiently small. In our particular problem, the validity zone is the region filled by the plasma and denoted by $\Omega_{P}(t)$ for $t>0$. The dynamics of the boundary of $\Omega_{P}(t)$ will be precised in Section 3.3.

\subsubsection{The one-fluid formulation of the quasi-neutral model}

In Ref. 10, concerned with the isentropic case, it has been showed that it is possible to write the quasi-neutral model with three different formulations: a constrained two-fluid formulation, a 1.5-fluid formulation and a one-fluid formulation. All these formulations are formally equivalent, but have different properties. The constrained two-fluid formulation is a strictly hyperbolic system, the 1.5-fluid formulation is a hyperbolic system and the one-fluid formulation is a conditionally hyperbolic system which is well-posed in one space dimension only. Although strict hyperbolicity is a better framework for numerical schemes, the numerical simulations of the plasma expansion test case, have shown that the 1.5-fluid formulations is the best formulation. The one-fluid formulation is as efficient as the 1.5-fluid formulation in the one-dimensional case but cannot be extended in dimensions more than 1 . Furthermore, it has been proved that the one-fluid formulation contains all the information in its hyperbolic part and then gives precious qualitative informations on the quasineutral solution. Indeed the hyperbolicity condition of this system corresponds to the condition of linear stability for the quasi-neutral model. Finally, in one space dimension the one-fluid formulation is simpler to discretize than (3.12)-(3.16), (3.8) and (3.19). Then in Section 5 we use this one-fluid formulation. We precise it bellow.

Due to quasi-neutrality, the electric potential variations are of the order of the Debye length. Then in the quasi-neutral limit, the potential is given by the anode 
potential and we do not need to calculate it. Following this idea, the one-fluid formulation is obtained eliminating the electric potential from the fluid equations. To do this, we subtract (3.13) and (3.14), this gives an expression for $\nabla \phi$ which we insert in the sum of (3.13) and (3.14) as well as in (3.15) and (3.16). We obtain

$$
\begin{aligned}
& n_{i}=n_{e}=n, \quad u_{i}=u_{e}+\frac{j}{n}, \\
& \nabla \cdot j=0 \\
& (1+\varepsilon) n \nabla \phi=-\varepsilon \partial_{t} j-\varepsilon \nabla\left(j \otimes u_{e}+u_{e} \otimes j+\frac{j \otimes j}{n}\right)+\nabla p_{e}-\varepsilon \nabla p_{i}, \\
& \partial_{t} n+\nabla \cdot\left(n u_{e}\right)=0 \\
& (1+\varepsilon)\left(\partial_{t}\left(n u_{e}\right)+\nabla\left(n u_{e} \otimes u_{e}\right)\right)+\nabla\left(j \otimes u_{e}+u_{e} \otimes j+\frac{j \otimes j}{n}\right) \\
& +\nabla\left(p_{i}+p_{e}\right)=-\partial_{t} j \\
& \partial_{t} w_{i}+\nabla \cdot\left(\left(w_{i}+p_{i}\right)\left(u_{e}+\frac{j}{n}\right)\right)+\frac{1}{1+\varepsilon}\left(u_{e}+\frac{j}{n}\right) \cdot\left(\nabla p_{e}-\varepsilon \nabla p_{i}\right) \\
& -\frac{\varepsilon}{1+\varepsilon}\left(u_{e}+\frac{j}{n}\right) \cdot \nabla\left(j \otimes u_{e}+u_{e} \otimes j+\frac{j \otimes j}{n}\right) \\
& =\frac{\varepsilon}{1+\varepsilon}\left(u_{e}+\frac{j}{n}\right) \cdot \partial_{t} j \\
& \partial_{t} w_{e}+\nabla \cdot\left(\left(w_{e}+p_{e}\right) u_{e}\right)-\frac{1}{1+\varepsilon} u_{e} \cdot\left(\nabla p_{e}-\varepsilon \nabla p_{i}\right) \\
& +\frac{\varepsilon}{1+\varepsilon} u_{e} \cdot \nabla\left(j \otimes u_{e}+u_{e} \otimes j+\frac{j \otimes j}{n}\right)=-\frac{\varepsilon}{1+\varepsilon} u_{e} \cdot \partial_{t} j,
\end{aligned}
$$

with the equations of state given by (3.8).

Note that this formulation is not conservative, so it can only be used for regular solutions without shocks. This is the case in our plasma expansion applications. Following this remark it is natural to write the total energy conservation equations (3.25) and (3.26) in terms of pressures, i.e.

$$
\begin{aligned}
& \partial_{t} p_{i}+\left(u_{e}+\frac{j}{n}\right) \cdot \nabla p_{i}+\gamma_{i} p_{i} \nabla \cdot\left(u_{e}+\frac{j}{n}\right)=0, \\
& \partial_{t} p_{e}+u_{e} \cdot \nabla p_{e}+\gamma_{e} p_{e} \nabla \cdot u_{e}=0 .
\end{aligned}
$$

In Section 3.2 we will see that the electric current is known in the beam region in the one-dimensional case. Then, the continuity of the current provides the current in the plasma region at the plasma-beam interface. Thus, in the one-dimensional case, Eq. (3.21) fully determines the current as a time dependent function and (3.22) determines the potential. In the two or three dimensional cases, this is more complicated. Indeed (3.21) is a scalar equation which cannot determine the current vector. It must be complemented by (3.22) in order to determine both the current vector and the scalar potential. For this reason, this one-fluid model is used only in one-dimensional case. 
In this case, the current, completely determined by the beam region, is a parameter of the system (3.20)-(3.26). And this system is conditionally hyperbolic. The hyperbolicity domain is characterized by

$$
\gamma_{e} p_{e}+\gamma_{i} p_{i} \geq \frac{\varepsilon j^{2}}{(1+\varepsilon) n} .
$$

We recall that, in Ref. 10, it has been proved, for isentropic pressure laws, that this hyperbolicity condition corresponds to the linear stability condition of the quasineutral model. Then, this condition gives the validity domain of the quasi-neutral model.

\subsubsection{Initial and boundary conditions for the quasi-neutral model}

Like for the rescaled two-fluid Euler-Poisson system we assume that the domain is initially devoid of plasma and that we inject a quasi-neutral plasma from the anode with the same velocities for the two species. Then, the anode boundary conditions for the quasi-neutral model are given by

$$
\left.n_{i}\right|_{x_{1}=0}=\left.n_{e}\right|_{x_{1}=0}=n_{0},\left.\quad u_{i}\right|_{x_{1}=0}=\left.u_{e}\right|_{x_{1}=0}=u_{0},\left.\quad T_{i}\right|_{x_{1}=0}=\left.T_{e}\right|_{x_{1}=0}=T_{0},
$$

where $n_{0}, u_{0}$ and $T_{0}$ are respectively the rescaled density, velocity and temperature of the injected plasma. We recall that (3.30) should be understood as follows: suppose we introduce a discretization of the quasi-neutral system by means of a Godunov scheme. Then, (3.30) will be used to compute the flux of the Riemann problem across the domain boundary.

In order to close the quasi-neutral model in the plasma region, we must precise the boundary conditions prescribed at the plasma beam interface. It is well known for hyperbolic systems that the number of boundary conditions depends on the outgoing characteristics. We recall that the plasma-beam interface position moves in the course of the time. We will see in Section 3.3 that it moves with the electron velocity: $u_{e}$. Thus, at the plasma-beam interface, the number of the eigenvalues lower than $u_{e}$ gives the number of the boundary conditions we must impose. In the hyperbolicity region, the eigenvalues are given by $u_{e}, u_{i}=u_{e}+j / n$ and $u_{ \pm}=u_{p} \pm c$ where $u_{p}$ and $c$ are the mean velocity and the sound speed in the plasma. They are given by

$$
u_{p}=u_{e}+\frac{j}{(1+\varepsilon) n}=\frac{\varepsilon u_{e}+u_{i}}{(1+\varepsilon)}, \quad c=\left(\frac{1}{(1+\varepsilon) n}\left(\gamma_{e} p_{e}+\gamma_{i} p_{i}-\frac{\varepsilon j^{2}}{(1+\varepsilon) n}\right)\right)^{1 / 2} .
$$

In the problem we are interested in, some ions are emitted from the plasma-beam interface. Therefore the current $j$ is positive. This gives $u_{i} \geq u_{e}$ and $u_{+} \geq u_{e}$ at the plasma-beam interface. Finally an easy calculation shows that if $\gamma_{e} p_{e}+\gamma_{i} p_{i} \in$ $\left[\varepsilon j^{2} /((1+\varepsilon) n), j^{2} / n\right]$ then $u_{-} \geq u_{e}$. In this case the interface is supersonic and we do not need to impose a boundary condition. But if $\gamma_{e} p_{e}+\gamma_{i} p_{i}>j^{2} / n$ then $u_{-}<u_{e}$ and we need to impose one boundary condition at the considered point. 
These boundary conditions are precised in Section 3.3 when we connect the quasineutral plasma region with the non quasi-neutral beam region.

\subsection{The Child-Langmuir model in the beam region}

We begin this section with the derivation of the Child-Langmuir model. This model is obtained from the Euler-Poisson system by a formal asymptotic limit. It possesses analytical solutions in the one-dimensional case (see Ref. 16). We briefly recall their expressions in Section 3.2.2.

\subsubsection{The formal derivation of the Child-Langmuir model}

Let $t>0$, we let $\Omega_{B}(t)=\Omega \backslash \Omega_{P}(t)$ be the beam region. In this region, there are no electrons and consequently $n_{e}=0$. The ions satisfy the rescaled Euler-Poisson system

$$
\begin{aligned}
& \partial_{t} n_{i}+\nabla \cdot\left(n_{i} u_{i}\right)=0, \\
& \partial_{t}\left(n_{i} u_{i}\right)+\nabla\left(n_{i} u_{i} \otimes u_{i}\right)+\nabla p_{i}=-n_{i} \nabla \phi, \\
& \partial_{t} w_{i}+\nabla \cdot\left(\left(w_{i}+p_{i}\right) u_{i}\right)=-n_{i} u_{i} \cdot \nabla \phi, \\
& -\lambda^{2} \Delta \phi=n_{i} .
\end{aligned}
$$

At the plasma-beam interface, $\Gamma(t)=\partial \Omega_{P}(t) \cap \partial \Omega_{B}(t)$, we assume the continuity of the potential and of the ion density and velocity. Then, the rescaled boundary conditions are given by $\left.\phi\right|_{\Gamma(t)}=0,\left.n_{i}\right|_{\Gamma(t)}=n_{i}^{-},\left.u_{i}\right|_{\Gamma(t)}=u_{i}^{-}$and $\left.\phi\right|_{x_{1}=1}=\phi_{L}=$ $e \bar{\phi}_{L} / k_{B} \bar{T}_{0}$, where $n_{i}^{-}$and $u_{i}^{-}$are respectively the ion density and velocity at the plasma-beam interface given by the quasi-neutral model.

In our applications, the cathode potential $\bar{\phi}_{L}<0$ is very large. Then, in the beam region, we expect ion velocities of the order of $\sqrt{e\left|\bar{\phi}_{L}\right| / m_{i}}$ and not of the order of $\sqrt{k_{B} \bar{T}_{0} / m_{i}}$ like in the quasi-neutral zone (see Section 3.1 for the rescaling). Similarly the potential energy is of the order of $e\left|\bar{\phi}_{L}\right|$ and not of $k_{B} \bar{T}_{0}$ like in the plasma region. We introduce the following scaling parameter

$$
\eta=\frac{k_{B} \bar{T}_{0}}{e\left|\bar{\phi}_{L}\right|}
$$

and the appropriate change of variables for the beam region is given by $\bar{\phi}=$ $\phi\left(k_{B} \bar{T}_{0} / e\right) /\left|\bar{\phi}_{L}\right|=\eta \phi$ and $\bar{u}_{i}=u_{i} \sqrt{k_{B} \bar{T}_{0} / m_{i}} / \sqrt{e\left|\bar{\phi}_{L}\right| / m_{i}}=\sqrt{\eta} u_{i}$. Furthermore, in Ref. 14 we performed numerical simulations of the two-fluid model. They show that the currents in the plasma and in the beam are of the same order. Then, we rescale the electron density in order to respect this property. This gives $\bar{n}_{i} \bar{u}_{i}=n_{i} u_{i}$ i.e. $\bar{n}_{i}=n_{i} / \sqrt{\eta}$. For convenience reasons, we write the energy conservation equation in terms of the pressure. Inserting this new scaling in the previous Euler-Poisson 
system, we obtain

$$
\begin{aligned}
& \sqrt{\eta} \partial_{t} \bar{n}_{i}+\nabla \cdot\left(\bar{n}_{i} \bar{u}_{i}\right)=0, \\
& \sqrt{\eta} \partial_{t}\left(\bar{n}_{i} \bar{u}_{i}\right)+\nabla\left(\bar{n}_{i} \bar{u}_{i} \otimes \bar{u}_{i}\right)+\sqrt{\eta} \nabla p_{i}=-\bar{n}_{i} \nabla \bar{\phi}, \\
& \sqrt{\eta} \partial_{t} p_{i}+\bar{u}_{i} \cdot \nabla p_{i}+\gamma_{i} p_{i} \nabla \cdot \bar{u}_{i}=0, \\
& -\alpha \Delta \bar{\phi}=\bar{n}_{i},
\end{aligned}
$$

where

$$
\alpha=\frac{\lambda^{2}}{\eta \sqrt{\eta}}=\frac{\epsilon_{0}\left|\bar{\phi}_{L}\right|^{3 / 2}}{\sqrt{e m_{i}} L^{2}} \frac{1}{N_{0} \sqrt{k_{B} \bar{T}_{0} / m_{i}}},
$$

is the ratio of the Child-Langmuir current in a device of length $L$ (see Ref. 4) to the typical plasma current. We recall that $\lambda$ and $\eta$ are respectively given by (3.7) and (3.32). Since these currents are of same order, we consider $\alpha$ as an order one parameter. Finally, the rescaled boundary conditions are given by

$$
\left.\bar{\phi}\right|_{\Gamma(t)}=0,\left.\quad \bar{n}_{i}\right|_{\Gamma(t)}=\frac{n_{i}^{-}}{\sqrt{\eta}},\left.\quad \bar{u}_{i}\right|_{\Gamma(t)}=\sqrt{\eta} u_{i}^{-} \text {and }\left.\quad \phi\right|_{x_{1}=1}=-1 .
$$

Now, the formal limit $\eta \rightarrow 0$ gives the Child-Langmuir problem, see Ref. 16:

$$
\left\{\begin{array}{l}
\nabla \cdot\left(\bar{n}_{i} \bar{u}_{i}\right)=0, \\
\nabla\left(\bar{n}_{i} \bar{u}_{i} \otimes \bar{u}_{i}\right)=-\bar{n}_{i} \nabla \bar{\phi}, \\
\bar{u}_{i} \cdot \nabla p_{i}+\gamma_{i} p_{i} \nabla \cdot \bar{u}_{i}=0, \\
-\alpha \Delta \bar{\phi}=\bar{n}_{i},
\end{array}\right.
$$

with the following boundary conditions

$$
\left.\bar{\phi}\right|_{\Gamma(t)}=0,\left.\quad \bar{u}_{i}\right|_{\Gamma(t)}=0,\left.\quad \bar{\phi}\right|_{x_{1}=1}=-1 .
$$

\subsubsection{The one-dimensional analytical solution of the Child-Langmuir model}

In Ref. 21 or Ref. 16, it has been showed that this system has an analytical solution in the one-dimensional case. Here, we recall briefly its expression. In one space dimension, the plasma-beam interface $\Gamma(t)$ is a point that we denote by $X(t)$. We set $\bar{j}_{i}=\bar{n}_{i} \bar{u}_{i}$ and we consider the case of an ion emission at the plasma-beam interface, so we assume $\bar{j}_{i}>0$. Then, system (3.34) leads to the fact that $\bar{j}_{i}$ does not depend on $x$ in $[X(t), 1]$.

Furthermore, we introduce the Child-Langmuir current:

$$
\bar{j}_{C L}(t)=4 \sqrt{2} \alpha /\left(9(1-X(t))^{2}\right),
$$

where we recall that $\alpha$ is given by (3.33). Then, there is no solution if $\bar{j}_{i} \notin\left[0, \bar{j}_{C L}(t)\right]$. But, for all values $\bar{j}_{i} \in\left[0, \bar{j}_{C L}(t)\right]$, there exists a unique solution $\left(\bar{n}_{i}, \bar{u}_{i}, \bar{\phi}\right)$ which is given, for all $x \in \Omega_{P}(t)=[X(t), 1]$ and all $t>0$, by

$$
\bar{n}_{i}(x, t)=\bar{j}_{i}(t) / \sqrt{-2 \bar{\phi}(x, t)}, \quad \bar{u}_{i}(x, t)=\sqrt{-2 \bar{\phi}(x, t)}, \quad p_{i}(x, t)=\bar{C}_{i} \bar{n}_{i}^{\gamma_{i}}(x, t),
$$


where $\bar{C}_{i}=p_{i}(X(t), t) / \bar{n}_{i}^{\gamma_{i}}(X(t), t)$ is unknown at this level.

The potential $\bar{\phi}$ is positive and implicitly determined by the relation

$$
\int_{0}^{\bar{\phi}(x, t)} \frac{d \psi}{\sqrt{\left(\bar{\phi}_{x}(X(t), t)\right)^{2}+2 \sqrt{2} \alpha^{-1} \bar{j}_{i}(t) \sqrt{-\psi}}}=X(t)-x,
$$

where the electric field at the plasma-beam interface $-\bar{\phi}_{x}(X)$ is calculated using the boundary condition $\left.\bar{\phi}\right|_{x=1}=1$ in the previous formula. In particular, for $\bar{j}_{i}=\bar{j}_{C L}(t)$, we have $\bar{\phi}_{x}(X)=0$ and

$$
\bar{\phi}(x, t)=-\left(\frac{x-X(t)}{1-X(t)}\right)^{4 / 3} .
$$

This concludes the presentation of the one-dimensional analytical solution of the Child-Langmuir model.

\subsection{The plasma-beam interface model}

Let us recall that there are two different systems for the description of the plasma bubble expansion. A quasi-neutral system for the plasma region and a ChildLangmuir system for the beam region. In order to close the model we must precise the dynamics of the plasma-beam interface and reconnect the quasi-neutral and the Child-Langmuir systems at this interface.

After rescaling the connection problem can be expressed in terms of the variable normal to the interface. Thus, the multi-dimensional case consists of onedimensional problems in the normal direction on each point of the plasma-beam interface. Consequently, we just describe the one-dimensional analysis and give the transmission relations in the multi-dimensional case. We reconnect the quasi-neutral model (3.20)-(3.24), (3.27), (3.28) with the Child-Langmuir model (3.37), (3.38). Following Ref. 13, this reconnection is obtained through the analysis of a onedimensional transmission problem. We begin with the evaluation of the different parameters that must be determined for the problem to be at least formally wellposed.

In the one-dimensional case, we recall that the plasma-beam interface reduces to a point. We denote it by $X(t)$. We must determine the time evolution of this point $X(t)$. Besides, in each zone some information is missing. Both the evolution of $X(t)$ and the missing information can be partially recovered by analyzing the transition problem.

At the plasma beam interface, we need to determine the current. We recall that, in the one-dimensional case, the current depends only on time in both zones. Then, it is sufficient to determine it at one point in the domain. Furthermore, in the beam zone, the current is known as soon as the electric field at the interface is known.

In the plasma region, we must impose some boundary conditions at the plasma beam interface for the conditionally hyperbolic system (3.23), (3.24), (3.27), (3.28) 
when it is subsonic. We have seen in Section 3.1 that if $\gamma_{e} p_{e}+\gamma_{i} p_{i} \in\left[\varepsilon j^{2} /((1+\right.$ $\left.\varepsilon) n), j^{2} / n\right]$, the interface is supersonic and we do not need to impose a boundary condition. But if $\gamma_{e} p_{e}+\gamma_{i} p_{i}>j^{2} / n$, we need to impose one boundary condition at the plasma beam interface.

Finally in the beam region, the determination of the pressure requires the knowledge of the ion density and pressure at the plasma beam interface.

Like in Ref. 13, we introduce a transmission problem in order to connect the two models. To this aim, we go back to the two-fluid Euler-Poisson system (3.1)-(3.6) and we zoom in on the plasma beam interface. Therefore, we introduce the change of variables $x \mapsto \xi=(x-X(t)) / \lambda$ and we set $f(x, t)=\tilde{f}(\xi, t)$ for all functions $f$. Omitting the "tildes" and formally passing to the limit $\lambda \rightarrow 0$, we obtain

$$
\begin{aligned}
& \partial_{\xi}\left(n_{i, e}\left(u_{i, e}-X^{\prime}\right)\right)=0, \\
& \partial_{\xi}\left(n_{i} u_{i}\left(u_{i}-X^{\prime}\right)+p_{i}\right)=-n_{i} \partial_{\xi} \phi, \\
& \partial_{\xi}\left(\varepsilon n_{e} u_{e}\left(u_{e}-X^{\prime}\right)+p_{e}\right)=n_{e} \partial_{\xi} \phi, \\
& \left(u_{i, e}-X^{\prime}\right) \partial_{\xi} p_{i, e}+\gamma_{i, e} p_{i, e} \partial_{\xi} u_{i, e}=0, \\
& -\partial_{\xi \xi}^{2} \phi=n_{i}-n_{e},
\end{aligned}
$$

where $X^{\prime}$ is the plasma-beam interface velocity.

Let us be more precise about the boundary conditions for this transmission problem. We are looking for solutions which connect the solution of the quasineutral system (3.20)-(3.24), (3.27), (3.28) at $\xi \rightarrow-\infty$, to the solution of the Child-Langmuir problem (3.37), (3.38) at $\xi \rightarrow \infty$. Furthermore, since the plasmabeam interface is located at $\xi=0$, we set $n_{e}=0$ for $\xi>0$. Finally we are looking for solutions such that $n_{e}$ is continuous at the plasma-beam interface $\xi=0$. Indeed, it is well known that for an ordinary fluid a shock wave cannot border the vacuum, see Ref. 28. The boundary conditions are the following for $\xi \rightarrow-\infty$ :

$$
n_{i}, n_{e} \rightarrow n_{-}, u_{i} \rightarrow u_{-}+\frac{j}{n_{-}}, u_{e} \rightarrow u_{-}, p_{i} \rightarrow p_{i-}, p_{e} \rightarrow p_{e-}, \phi, \phi_{\xi} \rightarrow 0,
$$

where $n_{-}=\left.n\right|_{X(t)-0}, u_{-}=\left.u_{e}\right|_{X(t)-0}, p_{i-}=\left.p_{i}\right|_{X(t)-0}, p_{e-}=\left.p_{e}\right|_{X(t)-0}$ are the values of the quasi-neutral system solution at the plasma-beam interface. Furthermore, $j$ is the current in the plasma region. We recall that it depends only on time.

For $\xi \rightarrow \infty, u_{i}$ must reconnect to the Child-Langmuir velocity, which is large of the order $O\left(\eta^{-1 / 2}\right)$, because of the change of scale in the beam region (see discussion at the beginning of Section 3.2). Therefore, for $\xi \rightarrow \infty, u_{i} \sim \eta^{-1 / 2}$ while $X^{\prime}$ is of order $O(1)$. We deduce that $n_{i}\left(u_{i}-X^{\prime}\right) \sim n_{i} u_{i}$ as $\xi \rightarrow \infty$ and the reconnection to the Child-Langmuir region implies that

$$
n_{i}\left(u_{i}-X^{\prime}\right) \rightarrow \bar{j}_{i}, \quad \text { when } \xi \rightarrow \infty,
$$

where $\bar{j}_{i}$ is the ion flux of the Child-Langmuir problem. Finally, we shall be looking for a solution such that $n_{i}$ is bounded as $\xi \rightarrow \infty$, otherwise the traveling-wave solution cannot be used to reconnect the two models. 
In Ref. 13, it is shown that non regular solutions (shock waves) cannot be solutions of this system in the isentropic case. But, we will see that solving this system amounts to considering isentropic pressure laws. Then, we assume regular solutions.

Thanks to (3.40) we have $n_{i, e}(\xi, t)\left(u_{i, e}(\xi, t)-X^{\prime}\right)=J_{i, e}(t)$ for all $\xi \in \mathbb{R}$ and all $t>0$, where the ion and electron current $J_{i}(t)$ and $J_{e}(t)$ are unknown at this level. Inserting this result in (3.43), this gives

$$
\frac{\partial_{\xi} p_{i, e}}{p_{i, e}}=-\gamma_{i, e} \frac{\partial_{\xi} u_{i, e}}{\left(u_{i, e}-X^{\prime}\right)}=-\gamma_{i, e} \frac{\partial_{\xi} J_{i, e} / n_{i, e}}{J_{i, e} / n_{i, e}}=\gamma_{i, e} \frac{\partial_{\xi} n_{i, e}}{n_{i, e}} .
$$

Integrating the previous equation, we obtain

$$
p_{i, e}(\xi, t)=\bar{C}_{i, e} n_{i, e}^{\gamma_{i, e}}(\xi, t),
$$

for $\xi \in \mathbb{R}, t>0$ and where $\bar{C}_{i, e}$ are constant parameters determined by the boundary conditions. Thus, the transmission problem (3.40)-(3.44) is formally equivalent to the following system:

$$
\begin{aligned}
& n_{i, e}\left(u_{i, e}-X^{\prime}\right)=J_{i, e}(t), \\
& \partial_{\xi}\left(n_{i} u_{i}\left(u_{i}-X^{\prime}\right)+p_{i}\right)=-n_{i} \partial_{\xi} \phi, \\
& \partial_{\xi}\left(\varepsilon n_{e} u_{e}\left(u_{e}-X^{\prime}\right)+p_{e}\right)=n_{e} \partial_{\xi} \phi, \\
& p_{i, e}=\bar{C}_{i, e} n_{i, e}^{\gamma_{i, e}} \\
& -\partial_{\xi \xi}^{2} \phi=n_{i}-n_{e} .
\end{aligned}
$$

We obtain the same interface problem as in the isentropic case solved in Ref. 13. We briefly recall its solution referring to Ref. 13 for details.

Since $n_{e}(\xi, t)=0$ for all $\xi>0$ and all $t>0$, Eq. (3.47) gives $J_{e}=0$. Then using the boundary conditions (3.45), we get

$$
X^{\prime}(t)=u_{-}=u_{e}(X(t), t),
$$

where $u_{e}$ is the electron velocity given by the quasi-neutral model (3.20)-(3.24), (3.27), (3.28).

Now, thanks to (3.47) and to the boundary conditions (3.45) and (3.46), we obtain the current continuity:

$$
j(t)=\bar{j}_{i}(t)
$$

where, we recall that $j$ is the current in the plasma zone and $j_{i}$ is the ion current in the beam region. Furthermore, the constant parameters in the pressure relations are given by

$$
\bar{C}_{i}=\frac{p_{i-}}{n_{-}^{\gamma_{i}}}=\frac{p_{i}(X(t), t)}{(n(X(t), t))^{\gamma_{i}}} \quad \text { and } \quad \bar{C}_{e}=\frac{p_{e-}}{n_{-}^{\gamma_{e}}}=\frac{p_{e}(X(t), t)}{(n(X(t), t))^{\gamma_{e}}}
$$

where $p_{i}, n$ and $p_{e}$ are given by the quasi-neutral model (3.20)-(3.24), (3.27), (3.28).

Finally using these results, in Ref. 13, we prove that the solution of (3.47)-(3.51) is obtained solving a differential system on the electric potential and the electric field (we refer to Ref. 13 for more details). A phase portrait analysis gives a necessary and 
sufficient condition for the existence of a solution to (3.40)-(3.44), (3.45) and (3.46). This condition is given by:

$$
\frac{j^{2}}{n_{-}} \geq \gamma_{e} p_{e-}+\gamma_{i} p_{i-} .
$$

This condition is nothing but the "Bohm sheath criterion" which is well-known in ion sheath physics (see Ref. 4, Ref. 23). Indeed this condition tells exactly that the interface, which moves at the electron velocity, must be supersonic, see the introduction at the beginning of this section.

This condition implicitly gives a boundary condition for the quasi-neutral model when it is necessary: if the interface is subsonic, that is if the density and the pressure in the plasma are such that $j^{2} / n_{-}<\gamma_{e} p_{e-}+\gamma_{i} p_{i-}$, then we impose the following condition at the plasma-beam interface:

$$
\frac{j(t)^{2}}{n(X(t), t)}=\gamma_{e} p_{e}(X(t), t)+\gamma_{i} p_{i}(X(t), t) .
$$

We will see in Section 4.2 that this condition allows for the existence of a shock wave between the plasma and the vacuum in the quasi-neutral model contrary to classical plasma-fluid interface problem for the two-fluid Euler-Poisson system, see Ref. 28. But, this shock cannot be too strong since the density in the plasma is limited by the condition (3.55).

Unfortunately, like in the isentropic case, this transmission problem gives the current continuity but not the value of this current. In order to assign a value to $\bar{j}_{i}=j$, we assume that the emission in the beam is the maximal possible current emission that is, $\phi_{x}(X(t), t)=0$ for all $t>0$ which gives

$$
j(t)=j_{C L},
$$

where $j_{C L}$ is the Child-Langmuir current defined by (3.36).

We generalize these transmission relations in the multi-dimensional case. First, following (3.52), the velocity of each point of the plasma-beam interface is given by the electron velocity. Then, for all $t>0$ and all $x(t) \in \Gamma(t)$

$$
\frac{d x(t)}{d t}=u_{e}(x(t), t)
$$

We assume the continuity of the current at the plasma-beam interface. Therefore Eq. (3.53) is generalized by $j(x(t), t)=\bar{j}_{i}(x(t), t)$ for all $t>0$ and all $x(t) \in \Gamma(t)$.

The pressure laws are still given by (3.54) changing $X(t)$ into $x(t)$. Furthermore the boundary condition for the quasi-neutral model at the plasma-beam interface is given by

$$
\frac{|j(x(t), t)|^{2}}{n(x(t), t)}=\gamma_{e} p_{e}(x(t), t)+\gamma_{i} p_{i}(x(t), t),
$$

if $|j(x(t), t)|^{2} / n(x(t), t)<\gamma_{e} p_{e}(x(t), t)+\gamma_{i} p_{i}(x(t), t)$, unless the interface is supersonic. 
Finally, assuming a maximal current regime in the beam, we consider the following boundary condition for the Child-Langmuir system (3.34)

$$
(\nabla \phi \cdot \nu)(x(t), t)=0,
$$

where $\nu$ is the unit normal to $\Gamma(t)$ outwards to $\Omega_{P}(t)$.

\section{Discretization of the one-dimensional asymptotic model}

First we summarize the asymptotic model in the one-dimensional case. It is constituted of two systems, one for the plasma region $\Omega_{P}(t)=[0, X(t)]$ and one for the beam region $\Omega_{B}(t)=[X(t), 1]$. The dynamics of the plasma-beam interface is given by (3.52) for all $t>0$.

The system for the plasma region consists of the one-fluid formulation of the quasi-neutral model (3.20)-(3.24), (3.27), (3.28). The current $j$ is given by (3.57). Initially the domain is supposed to be devoid of plasma and the boundary conditions are defined by (3.30) at the anode $x=0$. At the plasma-beam interface we impose (3.56) if the interface is subsonic, that is if $j^{2} / n(X(t), t)<$ $\gamma_{e} p_{e}(X(t), t)+\gamma_{i} p_{i}(X(t), t)$. In the beam region, we use the Child-Langmuir model given by (3.37), (3.39), (3.53) and (3.54).

Since the Child-Langmuir model has an explicit solution, the only points which deserve some discussion are the discretization of the motion of the plasma-beam interface (3.52) and that of the quasi-neutral model (3.20)-(3.24), (3.27), (3.28) in the plasma region $[0, X(t)]$ for all $t>0$.

We begin the presentation of the discretization with the description of the general principle of the scheme. Then, in Section 4.2, we calculate the approximate interface velocity with the help of a particular Riemann problem.

\subsection{General principle of the scheme}

The quasi-neutral model (3.20)-(3.24), (3.27), (3.28) can be written in the following form

$$
\partial_{t} W+A(W) \partial_{x} W=S(W)
$$

where $W=\left(n, u_{e}, p_{e}, p_{i}\right)^{T}, S(W)=\left(0,-\partial_{t} j /((1+\varepsilon) n), 0,0\right)^{T}$ and where $A$ is a four by four matrix defined by

$$
A(W)=\left(\begin{array}{cccc}
u_{e} & n & 0 & 0 \\
-j^{2} /\left((1+\varepsilon) n^{3}\right) & u_{e}+2 j /((1+\varepsilon) n) & 1 /((1+\varepsilon) n) & 1 /((1+\varepsilon) n) \\
0 & \gamma_{e} p_{e} & u_{e} & 0 \\
-\gamma_{i} p_{i} j / n^{2} & \gamma_{i} p_{i} & 0 & u_{e}+j / n
\end{array}\right) .
$$

We denote by $\Delta t>0$ the time step and we set $t_{m}=m \Delta t$ for all $m \in \mathbb{N}$. We refer to the m-th step as the step consisting in determining an approximation of the variable $W$ at time $t^{m+1}$ knowing its approximation at time $t^{m}$. 
We recall that the domain is defined by $[0, X(t)]$ and so it varies with time. Then, we use a space discretization which follows the interface position. We begin with a uniform discretization of $[0,1]$ such that $[0,1]=\cup_{k=1}^{N} M_{k}$ where $N \in \mathbb{N}^{*}$ is the given number of cells and $M_{k}=\left[x_{k-1 / 2}, x_{k+1 / 2}\left[\right.\right.$ with $x_{k-1 / 2}=(k-1) \Delta x$ and $\Delta x=1 / N$. During the m-th step, the interface position $X^{m}$ at time $t^{m}$ is known and the interface position $X^{m+1}$ at time $t^{m+1}$ is calculated with an explicit discretization of (3.52) given by

$$
X^{m+1}=X^{m}+\Delta t u_{I}^{m},
$$

where $u_{I}^{m}$ is an approximation of the interface velocity calculated in Section 4.2. In particular, we will see that $u_{I}^{m}$ is completely determined by the approximate values of $W$ at time $t^{m}$.

We denote by $k_{0}^{m}$ the index of the uniform cell $M_{k_{0}^{m}}$ containing $X^{m}$. Then, during the m-th step the mesh for the plasma region is defined by $\cup_{k=1}^{k_{0}^{m}-2} M_{k} \cup\left[x_{k_{0}^{m}-3 / 2}, X^{m}\right.$ [ at time $t^{m}$ and by $\cup_{k=1}^{k_{0}^{m}-2} M_{k} \cup\left[x_{k_{0}^{m}-3 / 2}, X^{m+1}\right.$ [ at time $t^{m+1}$, see Fig. 1 .

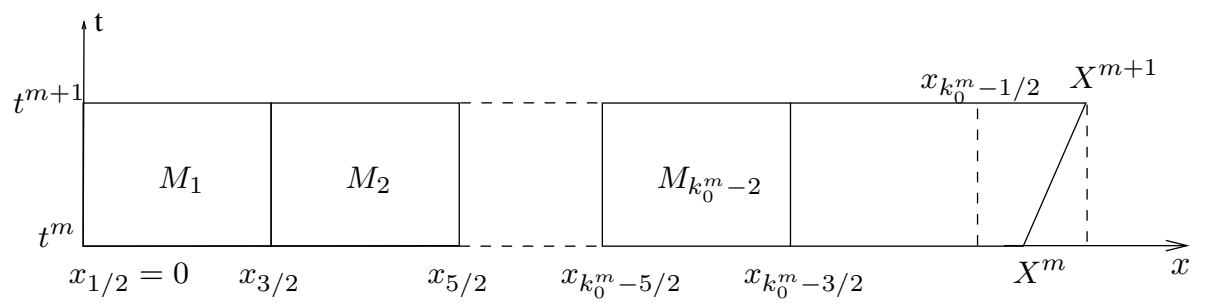

Fig. 1. Space discretization of the plasma zone at times $t^{m}$ and $t^{m+1}$ during the m-th step. The $\mathrm{m}$-th step consists in determining an approximation of the variable $W$ at time $t^{m+1}$ knowing this approximation at time $t^{m}$.

We choose to put together the cell $M_{k_{0}^{m}-1}$ and the interval $\left[x_{k_{0}^{m}-1 / 2}, X^{m}\right.$ [in order to avoid restrictive C.F.L. conditions. Indeed $X^{m}-x_{k_{0}^{m}-1 / 2}$ can be very small while $X^{m}-x_{k_{0}^{m}-3 / 2}$ is always bigger than $\Delta x$.

We consider a piecewise constant approximation, then we denote by $W_{k}^{m}$ an approximation of $W$ at time $t^{m}$ during the m-th step, on $M_{k}$ for $k=1, \cdots, k_{0}^{m}-2$ and on $\left[x_{k_{0}^{m}-3 / 2}, X^{m}\right.$ [ for $k=k_{0}^{m}-1$. We are looking for an approximation $W_{k}^{m+1}$ of $W$ at time $t^{m+1}$ during the m-th step, on $M_{k}$ for $k=1, \cdots, k_{0}^{m}-2$ and on $\left[x_{k_{0}^{m}-3 / 2}, X^{m+1}\left[\right.\right.$ for $k=k_{0}^{m}-1$.

First, we present the scheme for interior cells, i.e. for $k=1, \cdots, k_{0}^{m}-2$. We recall that we are interested in problems such that the solution in the plasma region is a regular function. Then, we can use the non conservative form of the hyperbolic system (4.1). We split the matrix $A$ in its positive and negative parts: $A(W)=$ $A^{+}(W)-A^{-}(W)$ where we recall that the matrix $A^{+}(W)$ is a diagonal matrix in the eigenvectors basis. Its diagonal is constituted of the maxima of 0 and of each eigenvalue of $A$. In practice we do not calculate $A^{+}$and $A^{-}$but following Ref. 15 , 
we use approximations of these matrices. It consists in writing $2 A^{+}=|A|+A$, $2 A^{-}=|A|-A$ and approximating $|A|$ with a polynomial function (here of degree 2 ) of the variable $A$. For more detail we refer to Ref. 15 or Ref. 10.

We denote by $A_{k, k-1}^{+, m}$ and by $A_{k+1, k}^{-, m}$, the respective polynomial approximations of $A^{+}\left(\left(W_{k}^{m}+W_{k-1}^{m}\right) / 2\right)$ and $A^{-}\left(\left(W_{k+1}^{m}+W_{k}^{m}\right) / 2\right)$. Then, the numerical scheme for interior cells $k=1, \cdots, k_{0}^{m}-2$, is given by

$$
\begin{aligned}
\Delta x\left(W_{k}^{m+1}-W_{k}^{m}\right)+\Delta t\left[A_{k, k-1}^{+, m}\left(W_{k}^{m}-W_{k-1}^{m}\right)-\right. & \left.A_{k+1, k}^{-, m}\left(W_{k+1}^{m}-W_{k}^{m}\right)\right] \\
& =\left(0,-\Delta x \frac{j^{m+1}-j^{m}}{(1+\varepsilon) n_{k}^{m+1}}, 0,0\right)^{T}
\end{aligned}
$$

where the approximations $j^{m}$ and $j^{m+1}$ of the plasma current at times $t^{m}$ and $t^{m+1}$ are given using (3.57), (3.36), $X^{m}$ and $X^{m+1}$.

For the last cell $\left[x_{k_{0}^{m}-3 / 2}, X^{m}\right.$ [, we introduce the solution of a Riemann problem. It consists in solving (4.1) without the source term and with a piecewise constant initial data $W(x, 0)=W_{k_{0}^{m}}^{m}$, if $x<0$, and $W(x, 0)=\left(0, u_{e, d}, p_{e, d}, p_{i, d}\right)^{T}$, if $x>0$. We denote by $W_{I}^{m}$ the value bordering the vacuum of this solution. This value is calculated in Section 4.2. And the scheme on the last cell is given by

$$
\begin{aligned}
& \frac{X^{m+1}-x_{k_{0}^{m}-3 / 2}}{\Delta t} W_{k_{0}^{m}-1}^{m+1}-\frac{X^{m}-x_{k_{0}^{m}-3 / 2}}{\Delta t} W_{k_{0}^{m}-1}^{m}-u_{I}^{m} W_{I}^{m}+ \\
& \left(\frac{X^{m+1}+X^{m}}{2}-x_{k_{0}^{m}-3 / 2}\right)\left[A_{k_{0}^{m}-1, k_{0}^{m}-2}^{+, m} \frac{W_{k_{0}^{m}-1}^{m}-W_{k_{0}^{m}-2}^{m}}{\Delta x}-A_{I}^{-, m} \frac{W_{I}^{m}-W_{k_{0}^{m}-1}^{m}}{X^{m}-x_{k_{0}^{m}-1}}\right] \\
& =\left(0,-\frac{X^{m+1}-x_{k_{0}^{m}-3 / 2}}{\Delta t} \frac{j^{m+1}-j^{m}}{(1+\varepsilon) n_{k_{0}^{m}-1}^{m+1}}, 0,0\right)^{T},
\end{aligned}
$$

where $A_{I}^{-, m}$ and $A_{k_{0}^{m}-1, k_{0}^{m}-2}^{+, m}$ are the polynomial approximations of $A^{+}\left(W_{I}^{m}\right)$ and of $A^{+}\left(\left(W_{k_{0}^{m}-1}^{m}+W_{k_{0}^{m}-2}^{m}\right) / 2\right)$.

In order to perform the next step, i.e. the $(\mathrm{m}+1)$-th step, we have to re-initiate the mesh for the plasma region and the associated approximation of $W$. To this aim, we consider the cell $M_{k_{0}^{m+1}}$ containing $X^{m+1}$. If $k_{0}^{m+1}=k_{0}^{m}$, the mesh and the associated approximation of $W$ are the same as in the m-th step. Otherwise $k_{0}^{m+1}=$ $k_{0}^{m}+1$ and the mesh is given by $\cup_{k=1}^{k_{0}^{m}-1} M_{k} \cup\left[x_{k_{0}^{m}-1 / 2}, X^{m+1}\left[=\cup_{k=1}^{k_{0}^{m+1}-2} M_{k} \cup\right.\right.$ $\left[x_{k_{0}^{m+1}-3 / 2}, X^{m+1}\left[\right.\right.$. The approximation of $W$ on the last cell $\left[x_{k_{0}^{m}-1 / 2}, X^{m+1}\right.$ [ is defined by setting $W_{k_{0}^{m+1}-1}^{m+1}=W_{k_{0}^{m+1}-2}^{m+1}=W_{k_{0}^{m}-1}^{m+1}$.

\subsection{A particular fluid-vacuum Riemann problem at the plasma-beam interface}

In order to precisely approximate the velocity of the plasma-beam interface position we introduce a particular Riemann problem. Since, in the beam region there is no plasma, the right state is the vacuum. But there are ions in the beam which exert 
a pressure. Therefore, this vacuum state has a non zero pressure. So, we consider the following Riemann problem:

$$
\left\{\begin{array}{l}
\partial_{t} W+A(W) \partial_{x} W=0, \\
W(x, 0)= \begin{cases}W_{g}=W_{k_{0}^{m}}^{m}=\left(n_{g}, u_{e, g}, p_{e, g}, p_{i, g}\right)^{T}, & \text { if } x<0, \\
W_{d}=\left(0, u_{e, d}, p_{e, d}, p_{i, d}\right)^{T}, & \text { if } x>0,\end{cases}
\end{array}\right.
$$

where $A$ is defined by (4.2).

It is well-known that the solution of this system is self-similar. We denote it by $\tilde{W}(x / t)=\left(\tilde{n}, \tilde{u}_{e}, \tilde{p}_{e}, \tilde{p}_{i}\right)^{T}$. We recall that we consider an ion emission at the plasma-beam interface. Then we assume $j(t)>0$ for all $t>0$. Furthermore, following the numerical results of the two-fluid Euler-Poisson model performed in Ref. 13, we are looking for a solution such that the state bordering the vacuum is separated from the vacuum by a shock wave or a contact discontinuity.

Now, we prove the following lemma:

Lemma 4.1. We consider the Riemann problem (4.3) and we assume $j(t)>0$ for all given $t>0$. We denote by $\tilde{W}(x / t)=\left(\tilde{n}, \tilde{u}_{e}, \tilde{p}_{e}, \tilde{p}_{i}\right)^{T}$ a solution to (4.3) such that the state bordering the vacuum and the vacuum are separated by a shock wave or a contact discontinuity.

Then, if $\gamma_{e} p_{e, g}+\gamma_{i} p_{i, g} \in\left[\varepsilon j^{2} /\left((1+\varepsilon) n_{g}\right), j^{2} / n_{g}\right]$, the state bordering the vacuum $W_{I}^{m}$ and the plasma beam interface velocity $u_{I}^{m}$ are respectively given by $W_{g}$ and $u_{e, g}$. But, if $\gamma_{e} p_{e, g}+\gamma_{i} p_{i, g}>j^{2} / n_{g}$, the state bordering the vacuum $W_{I}^{m}=\left(n_{I}, u_{e, I}, p_{e, I}, p_{i, I}\right)^{T}$ and the plasma beam interface velocity $u_{I}^{m}$ are given by

$$
\left\{\begin{array}{c}
p_{e, I}=C_{e} n_{I}^{\gamma_{e}}, \quad C_{i} n_{I}^{\gamma_{i}}, \quad \gamma_{e} p_{e, I}+\gamma_{i} p_{i, I}=\frac{j^{2}}{n_{I}} \\
u_{I}^{m}=u_{e, I}=u_{e, g}+\frac{j}{1+\varepsilon}\left(\frac{1}{n_{g}}-\frac{1}{n_{I}}\right) \\
-\int_{n_{g}}^{n_{I}} \frac{1}{s}\left(\frac{\gamma_{e} C_{e} s^{\gamma_{e}-1}+\gamma_{i} C_{i} s^{\gamma_{i}-1}}{(1+\varepsilon)}-\frac{\varepsilon j^{2}}{(1+\varepsilon)^{2} s^{2}}\right)^{1 / 2} d s
\end{array}\right.
$$

where $C_{e}=p_{e, g} / n_{g}^{\gamma_{e}}$ and $C_{i}=p_{i, g} / n_{g}^{\gamma_{i}}$.

Proof. We have seen in Section 3.1, that the system (4.3) is hyperbolic under the condition $\gamma_{e} p_{e}+\gamma_{i} p_{i}>\varepsilon j^{2} /((1+\varepsilon) n)$. In the hyperbolicity domain, this system has four eigenvalues given by $u_{e}, u_{i}=u_{e}+j / n, \mu_{+}=u_{p}+c$ and $\mu_{-}=u_{p}-c$ where $u_{p}$ and $c$ are given by (3.31).

Furthermore, if $\gamma_{e} p_{e}+\gamma_{i} p_{i} \in\left[\varepsilon j^{2} /((1+\varepsilon) n), j^{2} / n\right]$ all the eigenvalues of system (4.3) are bigger than $u_{e}$, see the discussion at the beginning of Section 3.3. But, thanks to (3.52), the plasma-beam interface velocity is given by $u_{e}$. Therefore, in a frame moving with the interface all the eigenvalues are positive. The associated characteristic fields are supersonic and no boundary conditions are necessary. 
But if $\gamma_{e} p_{e}+\gamma_{i} p_{i}>j^{2} / n$, one of the eigenvalues, $\mu_{-}$, is lower than $u_{e}$. The associated characteristic field is subsonic and we must impose a boundary condition. Following the analysis of the interface model, see Section 3.3, we impose the limit values satisfying the Bohm sheath criterion (3.56), that is $\gamma_{e} p_{e}+\gamma_{i} p_{i}=j^{2} / n$.

Before solving the Riemann problem (4.3), let us determine the nature of the elementary waves associated to each eigenvalues of $A$.

An easy calculation shows that the characteristic fields associated to $u_{e}$ and $u_{i}=$ $u_{e}+j / n$ are linearly degenerate. Then, the elementary waves, separating two states $W_{g}=\left(n_{g}, u_{e, g}, p_{e, g}, p_{i, g}\right)^{T}$ and $W_{d}=\left(n_{d}, u_{e, d}, p_{e, d}, p_{i, d}\right)^{T}$ and associated to $u_{e}$ or $u_{i}=u_{e}+j / n$, can only be contact discontinuities. Writing the Rankine-Hugoniot relations for the mass and total momentum conservation laws and denoting by $\sigma$ the wave velocity, we show that these contact discontinuities are characterized by

$$
\sigma=u_{e, g}=u_{e, d} \quad \text { and } \quad \frac{j^{2}}{n_{g}}+p_{e, g}+p_{i, g}=\frac{j^{2}}{n_{d}}+p_{e, d}+p_{i, d}
$$

for a contact discontinuity associated to $u_{e}$, and

$$
\sigma=u_{e, g}+\frac{j}{n_{g}}=u_{e, d}+\frac{j}{n_{d}} \quad \text { and } \quad-\varepsilon u_{e, g} j+p_{e, g}+p_{i, g}=-\varepsilon u_{e, d} j+p_{e, d}+p_{i, d}
$$

for a contact discontinuity associated to $u_{i}=u_{e}+j / n$.

Furthermore the characteristic fields associated to $\mu_{+}$and $\mu_{-}$are genuinely nonlinear. Then, the elementary waves, separating two states $W_{g}=\left(n_{g}, u_{e, g}, p_{e, g}, p_{i, g}\right)^{T}$ and $W_{d}\left(n_{d}, u_{e, d}, p_{e, d}, p_{i, d}\right)^{T}$ and associated to $\mu_{+}$or $\mu_{-}$, can be either shock waves or rarefaction waves.

Note that due to the non conservativity of the system (4.3), some RankineHugoniot relations are missing for the characterization of shock waves. But we will see that the Rankine-Hugoniot relations associated to the mass and total momentum conservation laws are sufficient to solve the particular fluid-vacuum Riemann problem (4.3).

For an elementary wave associated to $\mu_{+}$, we prove that:

If $n_{d} \geq n_{g}$, then, the states $W_{g}$ and $W_{d}$ can be connected by a $\mu_{+}$-rarefaction wave if and only if

$$
\frac{p_{e, g}}{n_{g}^{\gamma_{e}}}=\frac{p_{e, d}}{n_{d}^{\gamma_{e}}}, \quad \frac{p_{i, g}}{n_{g}^{\gamma_{i}}}=\frac{p_{i, d}}{n_{d}^{\gamma_{i}}} \quad \text { and } \quad Z_{+}\left(W_{g}\right)=Z_{+}\left(W_{d}\right)
$$

where for all $W=\left(n, u_{e}, p_{e}, p_{i}\right)^{T}$ in the hyperbolicity domain, $p_{e, i} / n^{\gamma_{e, i}}$ and $Z_{+}$are the Riemann invariants of the field $\mu_{+}$. The Riemann invariant $Z_{+}$is defined by

$$
Z_{+}(W)=u_{e}+\frac{j}{(1+\varepsilon) n}-\int_{n_{g}}^{n}\left(\frac{\gamma_{e} C_{e} s^{\gamma_{e}-1}+\gamma_{i} C_{i} s^{\gamma_{i}-1}}{(1+\varepsilon) s^{2}}-\frac{\varepsilon j^{2}}{(1+\varepsilon)^{2} s^{4}}\right)^{1 / 2} d s,
$$

with $C_{e}=p_{e, g} / n_{g}^{\gamma_{e}}$ and $C_{i}=p_{i, g} / n_{g}^{\gamma_{i}}$.

Otherwise, $W_{g}$ and $W_{d}$ can be connected by a $\mu_{+}$-shock wave if and only if

$$
\frac{p_{e, d}+p_{i, d}-\left(p_{e, g}+p_{i, g}\right)}{n_{d}-n_{g}} \geq \frac{\varepsilon j^{2}}{(1+\varepsilon) n_{d} n_{g}} \text {. }
$$


In this case, we write the Rankine-Hugoniot relations of the mass and total momentum conservation laws and the Lax conditions

$$
\mu_{+}\left(W_{g}\right)>\sigma_{+}>\mu_{+}\left(W_{d}\right) \quad \text { and } \quad u_{i}\left(W_{g}\right)<\sigma_{+},
$$

where $\sigma_{+}$is the velocity of the shock wave. This yields the $\mu_{+}$-shock wave characterization

$$
\begin{aligned}
& u_{e, g}=u_{e, d}+\frac{\left(n_{d}-n_{g}\right)\left(u_{e, d}-\sigma_{+}\right)}{n_{g}}, \\
& \sigma_{+}=u_{e, d}+\frac{1}{(1+\varepsilon) n_{d}}\left[j+\sqrt{-\varepsilon j^{2}+(1+\varepsilon) n_{d} n_{g} \frac{p_{e, d}+p_{i, d}-\left(p_{e, g}+p_{i, g}\right)}{n_{d}-n_{g}}}\right] .
\end{aligned}
$$

Similarly for an elementary wave associated to $\mu_{-}$, we prove that:

If $n_{d} \leq n_{g}$, then, the states $W_{g}$ and $W_{d}$ can be connected by a $\mu_{-}$-rarefaction wave if and only if

$$
\frac{p_{e, g}}{n_{g}^{\gamma_{e}}}=\frac{p_{e, d}}{n_{d}^{\gamma_{e}}}, \quad \frac{p_{i, g}}{n_{g}^{\gamma_{i}}}=\frac{p_{i, d}}{n_{d}^{\gamma_{i}}} \quad \text { and } \quad Z_{-}\left(W_{g}\right)=Z_{-}\left(W_{d}\right)
$$

where for all $W=\left(n, u_{e}, p_{e}, p_{i}\right)^{T}$ in the hyperbolicity domain, $Z_{-}$is defined by

$$
Z_{-}\left(n, u_{e}\right)=u_{e}+\frac{j}{(1+\varepsilon) n}+\int_{n_{g}}^{n}\left(\frac{\gamma_{e} C_{e} s^{\gamma_{e}-1}+\gamma_{i} C_{i} s^{\gamma_{i}-1}}{(1+\varepsilon) s^{2}}-\frac{\varepsilon j^{2}}{(1+\varepsilon)^{2} s^{4}}\right)^{1 / 2} d s .
$$

Otherwise, the states $W_{g}$ and $W_{d}$ can be connected by a $\mu_{-}$-shock wave if and only if (4.5) is satisfied. Then, using the Rankine-Hugoniot relations of the mass and total momentum conservation laws as well as the Lax conditions, we prove that a $\mu_{-}$-shock wave is characterized by

$u_{e, g}=u_{e, d}+\frac{\left(n_{d}-n_{g}\right)\left(u_{e, d}-\sigma_{-}\right)}{n_{g}}$,

$\sigma_{-}=u_{e, d}+\frac{1}{(1+\varepsilon) n_{d}}\left[j-\sqrt{-\varepsilon j^{2}+(1+\varepsilon) n_{d} n_{g} \frac{p_{e, d}+p_{i, d}-\left(p_{e, g}+p_{i, g}\right)}{n_{d}-n_{g}}}\right]$,

where $\sigma_{-}$is the velocity of the shock wave and where the Lax conditions are given by

$$
\mu_{-}\left(W_{g}\right)>\sigma_{-}>\mu_{-}\left(W_{d}\right) \quad \text { and } \quad u_{e}\left(W_{d}\right)>\sigma_{-} .
$$

Now, let us turn to the resolution of the Riemann problem (4.3). We recall that we are looking for a solution to (4.3) such that the state bordering the vacuum, denoted by $W_{I}=\left(n_{I}, u_{e, I}, p_{e, I}, p_{i, I}\right)^{T}$, and the vacuum are separated by a shock wave or a contact discontinuity. Then, writing the conservation of the mass, we obtain

$$
\left[n\left(u_{e}-\sigma\right)\right]=0 \quad \Leftrightarrow \quad n_{I}\left(u_{e, I}-\sigma_{I}\right)=0\left(u_{e, d}-\sigma_{I}\right) \quad \Leftrightarrow \quad u_{e, I}=\sigma_{I}
$$

where $\sigma_{I}$ is the velocity of the wave and also the velocity of the plasma-beam interface. 
We begin assuming $\gamma_{e} p_{e, g}+\gamma_{i} p_{i, g} \leq j^{2} / n_{g}$. In this case $u_{e g} \leq \mu_{-}\left(W_{g}\right) \leq$ $\mu_{+}\left(W_{g}\right) \leq u_{i, g}$, then all the waves are faster than the plasma beam interface velocity. The solution is given by the left state $W_{g}$ and the vacuum connected by a $u_{e}$ contact discontinuity and $W_{I}=W_{g}$.

Now, we assume that $\gamma_{e} p_{e, g}+\gamma_{i} p_{i, g}>j^{2} / n_{g}$, in this case the interface is subsonic: one of the eigenvalues, $\mu_{-}$is lower than the plasma-beam interface velocity, $u_{e}$. It is necessary to impose a boundary condition, and we recall we chose to assume (3.56). Using the characterizations of the elementary waves, we show that the solution is given by the left state $W_{g}$ connected to $W_{I}$ by a $\mu_{-}$rarefaction wave and the intermediate state $W_{I}$ connected to the vacuum by a $u_{e}$ contact discontinuity. Then, using the Riemann invariants relations (4.6), we obtain that $W_{I}$ is given by (4.4) $\square$

\section{Numerical results}

In order to validate the asymptotic model, we compare it to the Euler-Poisson system (3.1)-(3.6) in the one-dimensional case for two different test cases. In both cases we consider a quasi-neutral plasma expansion in the vacuum separating two electrodes. But in the first test case the plasma is injected from the anode while in the second test case it is injected from the cathode.

The discretization of the Euler-Poisson system (3.1)-(3.6) is standard, we use an explicit finite volume scheme with a Godunov solver for the hydrodynamic fluxes, a finite difference scheme for the Poisson equation and an implicit treatment for the source terms.

In the first test case, related to high current injectors for ions accelerators, we inject a quasi-neutral plasma from the anode located at $x=0$. We consider a plasma constituted of electrons and of Deuterium ions. Therefore $m_{i}=3,37 \cdot 10^{-27}$ $\mathrm{kg}$. Furthermore the physical parameters are the following, the ratios of specific heats are given by $\gamma_{i}=\gamma_{e}=\gamma=5 / 3$. The velocity, density and temperatures of the injected plasma are $N_{i}=N_{e}=N_{0}=10^{20} \mathrm{~m}^{-3}, U_{i}=U_{e}=U_{0}=3 \cdot 10^{4} \mathrm{~m} / \mathrm{s}$ and $\bar{T}_{i}=\bar{T}_{e}=5 \mathrm{eV}$. The length of the diode is $L=1,15 \cdot 10^{-2} \mathrm{~m}$ and the potential at the electrodes are: $\Phi_{K}=-10^{5}$ Volts for the cathode and $\Phi_{A}=0$ Volt for the anode. This yields the following scaled parameters

$$
\varepsilon=2,7 \cdot 10^{-4}, \quad \eta=5 \cdot 10^{-5} \text { and } \lambda=4,2 \cdot 10^{-4} .
$$

We use a uniform mesh with 7000 cells for the Euler-Poisson system and with 2000 cells for the asymptotic model. It is important to note that, even if the plasma period is resolved i.e. if Eq. (2.7) is satisfied, this is not possible to choose a coarse mesh for the Euler-Poisson system because some numerical instabilities appear at the plasma-beam interface. Furthermore, the computational time for the asymptotic model is 70 seconds while that of the Euler-Poisson model is more than 12 hours. Then there is a very important time saving using the asymptotic model. Figs. 2, 3, 4 and 5 give the results for this test case.

Fig. 2 (left) shows the quasi-neutral density in the plasma. The results are computed with the two-fluid Euler-Poisson and the quasi-neutral models. We note a 
good agreement of the curves at the beginning of the plasma expansion, but with a discrepancy between the results at the end of the simulation. This discrepancy manifests itself on the plasma-beam interface position, which the quasi-neutral model computes, a little bit ahead of that of the Euler-Poisson model and on the particle density which is too large around the anode for the quasi-neutral model. These problems certainly result from the presence of a boundary layer at the injection point. They were already observed in the isentropic case (see Ref. 13) but it seems that they are magnified when the energy conservation laws are considered. The detailed study of this boundary layer is a difficult problem and is in progress. It should also be noted that the electric field in the beam region is very large when the plasma-beam interface reaches the cathode, see Fig. 5 (right). Therefore, the current is very large and the problem becomes unstable and very difficult to solve. The physical relevance of the model is also questionable because then, the external circuit will limit the value of the current.

The same problems can be observed on the particle velocities in the plasma, see Fig. 3, and on the ion and electron pressures, see Fig. 4. Note that there are large oscillations around the interface on the electron velocity curve given by the Euler-Poisson model. These oscillations give large negative values for the electron velocity. We must cut them otherwise it is not possible to see the variations of the velocity in the plasma. These oscillations are related to the small mass ratio $\varepsilon$ present in the electron momentum conservation law.

On Fig. 2 (right), we plot the current inside the domain, computed with both the Euler-Poisson model and with the Child-Langmuir law. Once again, there is a good agreement at the beginning of the simulation and a discrepancy at the end of the process. This discrepancy is related with the error made for the determination of the interface since the Child-Langmuir depends on this position through (3.36). At the end of the simulation, the plasma-beam interface is no more well predicted by the asymptotic model and the current is consequently badly predicted too. The same remark can be made on the electric potential in the beam region given by Fig. 5. Note that the two-fluid Euler-Poisson model yields a non constant current for times close to the initial one (see the curve at the rescaled time $t=0.01$ ). This is due to the fact that the Child-Langmuir regime is not yet established. At large times, the Euler-Poisson model yields a constant current except at the plasma-beam interface where there is a peak. This peak, already present in the isentropic case (see Ref. 13), can be explained by means of the transmission problem solution. Indeed, in the transmission region, we have $j_{i}=n_{i}\left(u_{i}-d X(t) / d t\right)$ and $n_{e}\left(u_{e}-d X(t) / d t\right)=0$ where $j_{i}$ does not depend on $x$. But the current $j$ is given by $j=n_{i} u_{i}-n_{e} u_{e}=j_{i}+\left(n_{i}-n_{e}\right) d X(t) / d t$. Thus $j$ varies like $n_{i}-n_{e}$ and its shape, as a function of $x$, is exactly that of the observed peak. Note again that due to the large electric field in the beam region, the current is very large at the end of the simulation. And, the physical relevance of the model is questionable because in practice, the external circuit limits the value of the current.

In the second test case, related to the study of high current diodes and of arc 

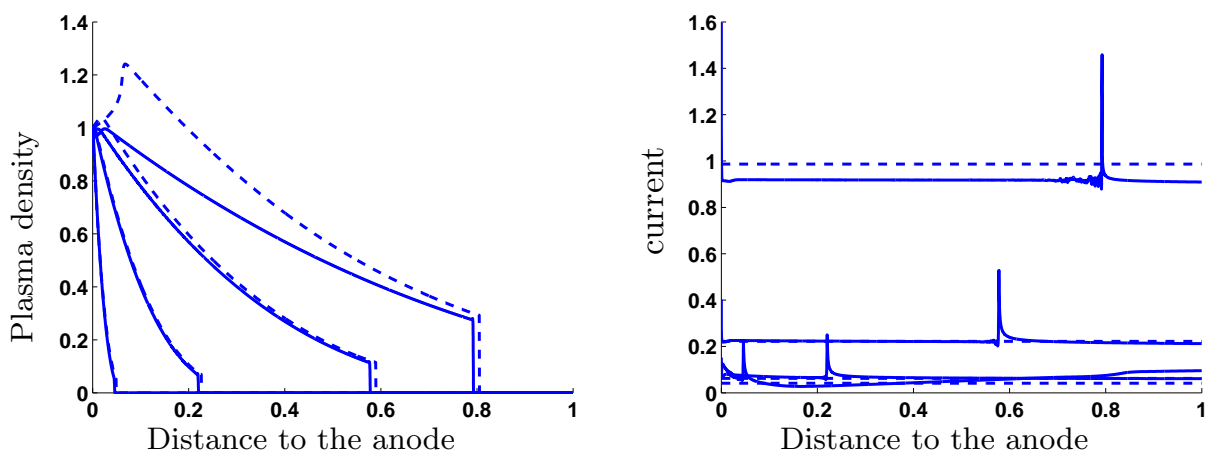

Fig. 2. Test case 1. Left: quasi-neutral density in the plasma, given by the Euler-Poisson model (solid line) and by the quasi-neutral model (dotted line). The results are given at the rescaled times $0.01,0.05,0.15$ and 0.3. Right: current inside the domain, given by the Euler-Poisson model (solid line) and by the Child-Langmuir model (3.36) using the plasma-beam interface calculated with the quasi-neutral model (dotted line). The results are given at the rescaled times 0.01, 0.05, 0.15 and 0.3 .
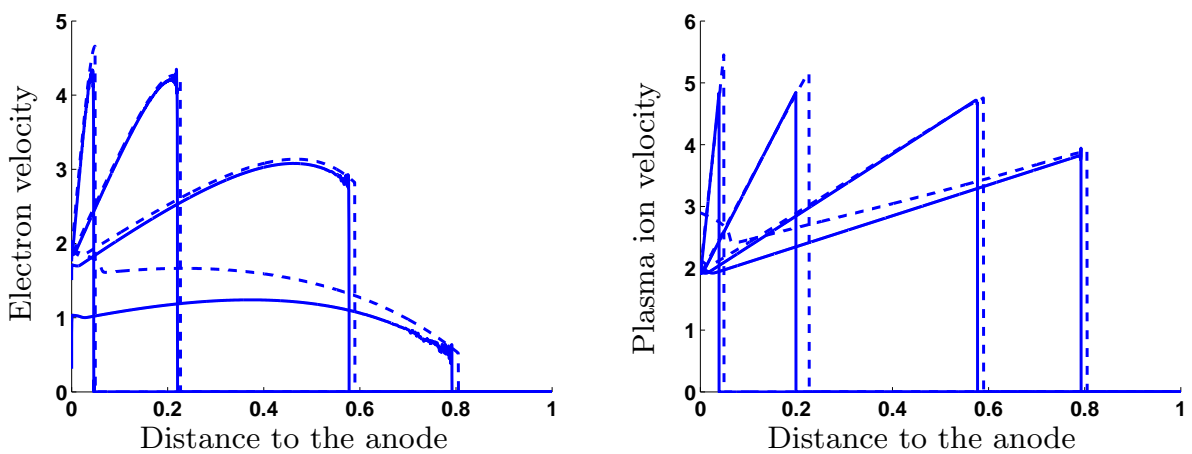

Fig. 3. Test case 1. Left: positive part of the electron velocity given by the Euler-Poisson model (solid line) and electron velocity given by the quasi-neutral model (dotted line) in the plasma region. The results are given at the rescaled times $0.01,0.05,0.15$ and 0.3 . Right: ion velocity given by the Euler-Poisson model (solid line) and by the quasi-neutral model (dotted line) in the plasma region at the rescaled times $0.01,0.05,0.15$ and 0.3 .

phenomena on satellites, the quasi-neutral plasma is injected from the cathode located at $x=0$. Then the emitted particles from the plasma-beam interface are now electrons. We consider the following rescaled parameters $\lambda=3,1 \cdot 10^{-4}, \varepsilon=10^{-4}$ and $\eta=10^{-3}$. We use a uniform mesh with 3500 cells for the Euler-Poisson system and with 1000 cells for the asymptotic model. Furthermore, the computational time for the asymptotic model is 34 seconds while that for the Euler-Poisson model is more than 3 hours. Then there is still an important time saving using the asymptotic model. Figs. 6, 7, 8 and 9 give the results for this test case. 

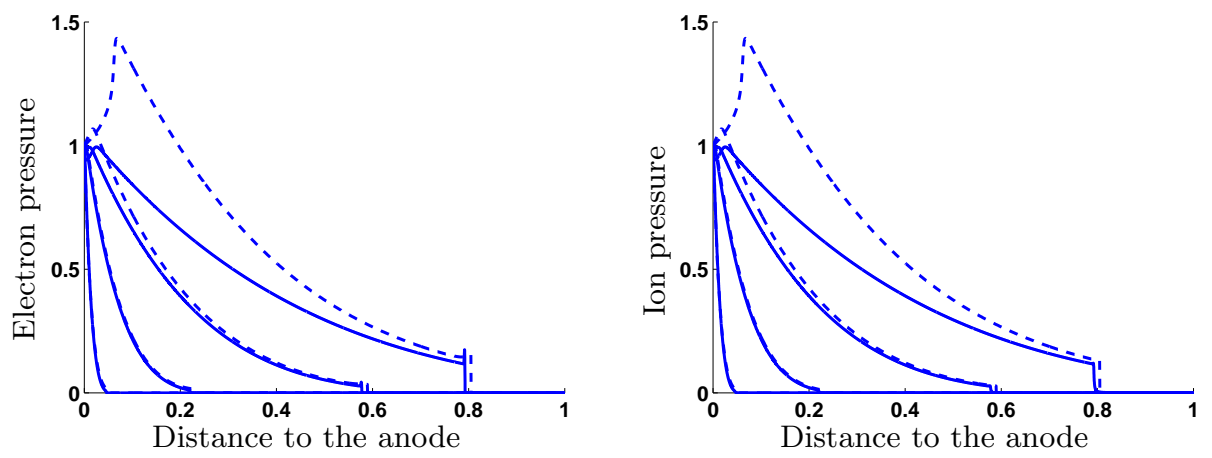

Fig. 4. Test case 1. Electron and ion pressures given by the Euler-Poisson model (solid line) and by the quasi-neutral model (dotted line) in the plasma region at the rescaled times $0.01,0.05,0.15$ and 0.3 .
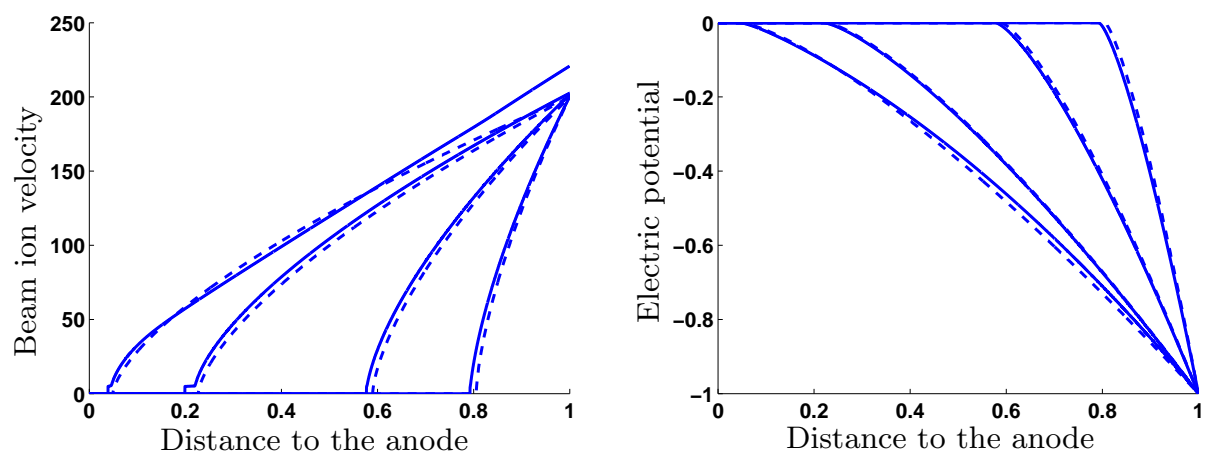

Fig. 5. Test case 1. Ion velocity and electric potential in the beam region given by the Euler-Poisson model (solid line) and by the Child-Langmuir model (dotted line) at the rescaled times $0.01,0.05$, 0.15 and 0.3 .

The results have overall an identical behavior compared with the results of the previous test case. The plasma-beam interface is well approximated during all the simulation (see Fig. 6). We still observe the presence of a boundary layer at the injection point, the cathode $x=0$, which generates an error on the results that increases with time. Remark that the comparison of the results stops earlier in the expansion process. This is due to the fact that we cannot give results for larger times for the asymptotic model. Just after the rescaled time $t=0.6$ the hyperbolicity of the quasi-neutral model is lost and then the simulation of the asymptotic model stops. In the case of an electron beam, the hyperbolicity region of the quasi-neutral model is characterized by

$$
\gamma_{e} p_{e}+\gamma_{i} p_{i} \geq \frac{\varepsilon j^{2}}{(1+\varepsilon) n}
$$


We recall that in order to have a solution to the transmission problem, a necessary and sufficient condition is that the interface is supersonic, which is characterized by

$$
\gamma_{e} p_{e}+\gamma_{i} p_{i} \leq \frac{\varepsilon j^{2}}{n}
$$

The smallness of $\varepsilon$ gives a very small range between the critical values $\varepsilon j^{2} /((1+\varepsilon) n)$ and $\varepsilon j^{2} / n$. This yields an unstable numerical resolution of this problem and explains why, contrary to the previous test case, we lose quite quickly the hyperbolicity of the quasi-neutral model. In Ref. 10, it has been showed that this breakdown of the model can be attributed to a physical two-stream instability. This instability occurs when the plasma current reaches large values as the interface moves closer to the anode. In this case, non quasi-neutral modes are excited and the quasi-neutral model fails to describe them. For these particular situations, the problem can be cured by using the Euler-Poisson model which describes both quasi-neutral and non quasi-neutral modes but it is necessary to discretize it with an asymptotic preserving scheme in the quasi-neutral limit. Such a scheme has been developed in the isentropic case, see Ref. 11. Investigation of this scheme for the full Euler case is in progress.

Furthermore, at the end of the simulation we remark a stronger discrepancy between the results of the two models in the beam region as compared to the previous test case. But, here the potential difference between the electrodes is one hundred times smaller than that applied in the previous test case. Thus it would certainly be more accurate to couple the quasi-neutral model with a one-fluid EulerPoisson model for the description of the beam instead of a child-Langmuir law.
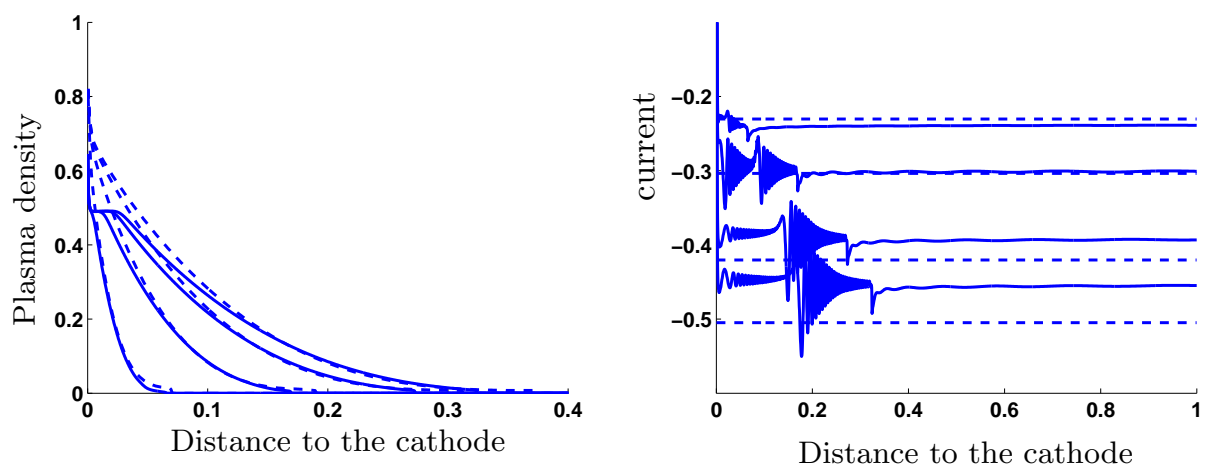

Fig. 6. Test case 2. Left: quasi-neutral density in the plasma, given by the Euler-Poisson model (solid line) and by the quasi-neutral model (dotted line). The results are given at the rescaled times 0.01, 0.03, 0.05 and 0.06. Right: current inside the domain, given by the Euler-Poisson model (solid line) and by the Child-Langmuir model (3.36) using the plasma-beam interface calculated with the quasi-neutral model (dotted line). The results are given at the rescaled times $0.01,0.03,0.05$ and 0.06 . 

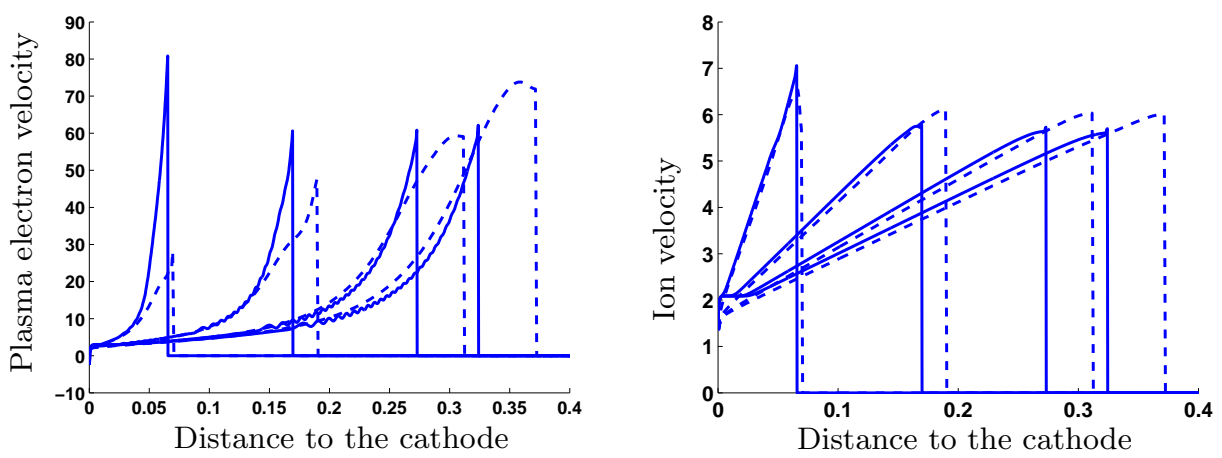

Fig. 7. Test case 2. Electron and ion velocities given by the Euler-Poisson model (solid line) and by the quasi-neutral model (dotted line) in the plasma region at the rescaled times $0.01,0.03,0.05$ and 0.06 .

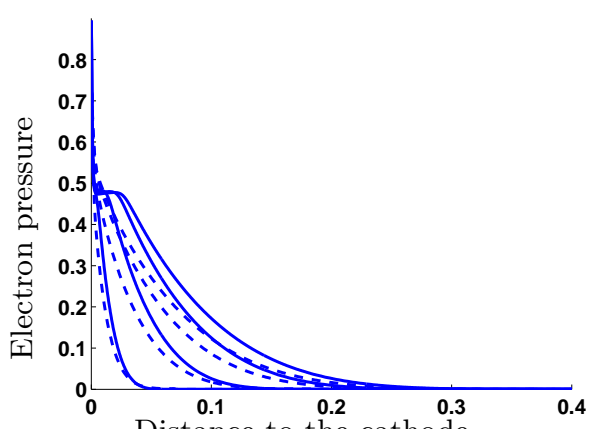

Distance to the cathode

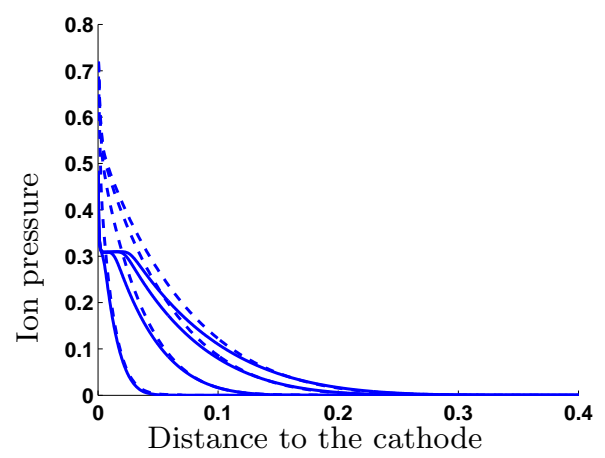

Distance to the cathode

Fig. 8. Test case 2. Electron and ion pressures given by the Euler-Poisson model (solid line) and by the quasi-neutral model (dotted line) in the plasma region at the rescaled times $0.01,0.03,0.05$ and 0.06 .
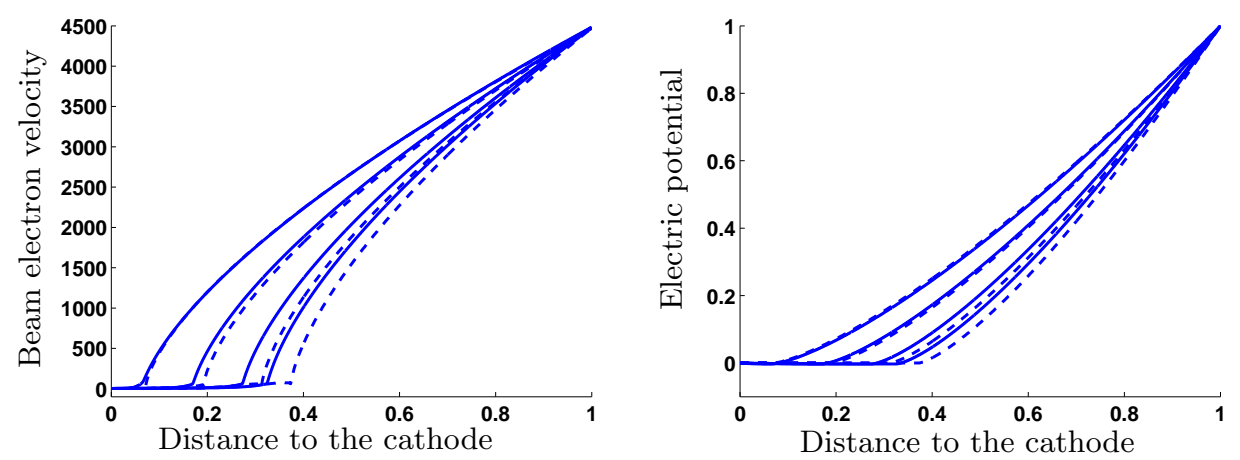

Fig. 9. Test case 2. Electron velocity and electric potential in the beam region given by the EulerPoisson model (solid line) and by the Child-Langmuir model (dotted line) at the rescaled times $0.01,0.03,0.05$ and 0.06 . 


\section{Conclusion}

In this paper, we have proposed a model for the description of a quasi-neutral plasma expansion between two electrodes. This model is obtained using an asymptotic analysis from the full-Euler-Poisson system. It consists of a quasi-neutral model for the plasma region and of a Child-Langmuir law for the beam region. They are coupled at the plasma-beam interface using transmission relations. These relations have been obtained from the study of a travelling wave problem in the transition region. Numerical simulations have been performed for two test cases in one space dimension. They confirm that the model produces a good approximation of the original two-fluid Euler-Poisson model and show that there is a very important time saving using the asymptotic model.

\section{References}

1. N. Ben Abdallah, The Child-Langmuir regime for electron transport in a plasma including a background of positive ions, Math. Models Methods Appl. Sci. 4 (1994) 409-438.

2. R. L. Boxman, P. J. Martin and D. M. Sanders (eds.), Handbook of vacuum arcs science and technology. Fundamentals and applications, (Noyes Publications, Park Ridge, 1995).

3. J-P. Catani and D. Payan, Electrostatic behaviour of materials in a charging space environment, Proceedings of the 9th International Symposium on Materials in a Space Environment, 16-20 June 2003, Noordwijk, The Netherlands (ESA Publications Division 2003).

4. F. F. Chen, Introduction to plasma physics (Plenum, New-York, 1974).

5. H-H. Choe, N. S. Yoon, S. S. Kim and D-I. Choi, A New Unconditionally Stable Algorithm for Steady-State Fluid Simulation of High Density Plasma Discharge, J. Comput. Phys. 170 (2001) 550-561.

6. M. Cho M and D. E. Hastings, Dielectric charging process and arcing rates of high voltage solar arrays, J. Spacecraft and Rockets 28 (1990) 698-706.

7. P. Colella, M. R. Dorr and D. D. Wake, A Conservative Finite Difference Method for the Numerical Solution of Plasma Fluid Equations, J. Comput. Phys. 149 (1999) $168-193$

8. S. Cordier and E. Grenier, Quasineutral limit of Euler-Poisson system arising from plasma physics, Comm. Partial Differential Equations 25 (2000) 1099-1113.

9. P. Crispel, Etude de la transition de la decharge primaire vers l'arc secondaire sur un générateur solaire de satellite, Phd thesis, SUPAERO, Toulouse, December 2005.

10. P. Crispel, P. Degond and M-H. Vignal, Quasi-neutral fluid models for current carrying plasmas, J. Comp. Phys 205 (2005) 408-438.

11. P. Crispel, P. Degond and M-H. Vignal, An asymptotic preserving scheme for the twofluid Euler-Poisson model in the quasi-neutral limit, J. Comput. Phys., to appear.

12. P. Degond, S. Jaffard, F. Poupaud and P.A. Raviart, The Child-Langmuir asymptotics of the Vlasov-Poisson equation for cylindrically or spherically symmetric diodes, part 1: statement of the problem and basic estimates, Math. Methods Appl. Sci.19 (1996) 287-312.

13. P. Degond, C. Parzani and M-H. Vignal, Plasma expansion in vacuum: modeling the breakdown of quasineutrality, SIAM Multiscale Modeling and Simulation 2 (2003) $158-178$. 
14. P. Degond, C. Parzani and M-H. Vignal, On plasma expansion in vacuum, in: Free Boundary Problems: Theory and Applications, P. Colli, C. Verdi and A. Visintin, eds., International Series of Numerical Mathematics, 147 (Birkhäuser Verlag, Basel, 2004).

15. P. Degond, P. F. Peyrard, G. Russo and Ph. Villedieu, Polynomial upwind schemes for hyperbolic systems, C. R. Acad. Sci. Paris, Ser. I 328 (1999) 479-483.

16. P. Degond and P. A. Raviart, An Asymptotic Analysis of the One-Dimensional VlasovPoisson System : the Child-Langmuir Law, Asymptotic Analysis 4 (1991) 187-214.

17. P. Degond, R. Talaalout and M. H. Vignal, Electron transport and secondary emission in a surface of solar cell, Proceeding of the Workshop 'Multipactor, RF and DC corona and passive intermodulation in space RF hardware', 4-6 September 2000, ESTEC, Noordwijk, The Netherlands.

18. P.O. Demchenko and M.G. Shulika, Ion beam forming dynamics in an injector taking into account a plasma boundary., Problems of atomic science and technology 3 (2001) 144-146.

19. S. Fabre, Stability analysis of the Euler-poisson equations, J. Comp. Phys. 101 (1992) 445-451.

20. N. A. Krall and A. W. Trivelpiece, Principles of plasma physics (San Francisco Press, 1986).

21. I. Langmuir and K. T. Compton, Electrical discharges in gases, part II, fundamental phenomena in electrical discharges, Rev. Modern Phys. 3 (1931) 191-257.

22. Y.-J. Peng and Y.-G. Wang, Boundary layers and quasi-neutral limit in steady state Euler-Poisson equations for potential flows, Nonlinearity, 173 (2004) 835-849.

23. K. U. Riemann, The Bohm criterion and sheath formation, J. Phys. D: Appl. Phys. 24 (1991) 493-518.

24. R. Schneider and C-D. Munz, The approximation of two-fluid plasma flow with explicit upwind schemes, Int. J. Numer. Model. 8 (2005) 399-416.

25. U. Shumlak and J. Loverich, Approximate Riemann solver for the two-fluid plasma model, J. Comp. Phys. 187 (2003) 620-638.

26. M. Slemrod and N. Sternberg, Quasi-neutral limit for Euler-Poisson system, J. Nonlinear Sci. 11 (2001) 193-209.

27. H. Sze, J. Benford, W. Woo and B. Harteneck, Dynamics of a virtual cathode oscillator driven by a pinched diode, Phys. Fluids 29 (1986) 3873-3880.

28. E.F. Toro, Riemann Solvers and Numerical Methods for Fluid Dynamics, Springer.

29. S. Wang, Quasineutral limit of Euler-Poisson system with and without viscosity, Comm. Partial Differential Equations, 29 (2004) 419-456. 